\title{
Roaming form factors for the tricritical to critical Ising flow
}

\author{
D.X. Horváth, ${ }^{a, b}$ P.E. Dorey ${ }^{c}$ and G. Takács ${ }^{a, b}$ \\ a MTA-BME "Momentum" Statistical Field Theory Research Group, \\ 1111 Budapest, Budafoki út 8, Hungary \\ ${ }^{b}$ Department of Theoretical Physics, Budapest University of Technology and Economics, \\ 1111 Budapest, Budafoki út 8, Hungary \\ ${ }^{c}$ Department of Mathematical Sciences, Durham University, \\ South Road, Durham DH1 3LE, U.K. \\ E-mail: esoxluciuslinne@gmail.com, p.e.dorey@durham.ac.uk, \\ takacsg@eik.bme.hu
}

ABStract: We study the massless flows described by the staircase model introduced by Al.B. Zamolodchikov through the analytic continuation of the sinh-Gordon S-matrix, focusing on the renormalisation group flow from the tricritical to the critical Ising model. We show that the properly defined roaming limits of certain sinh-Gordon form factors are identical to the form factors of the order and disorder operators for the massless flow. As a by-product, we also construct form factors for a semi-local field in the sinh-Gordon model, which can be associated with the twist field in the ultraviolet limiting free massless bosonic theory.

Keywords: Field Theories in Lower Dimensions, Integrable Field Theories

ARXiv EPrint: 1604.05635 


\section{Contents}

$\begin{array}{llr}1 & \text { Introduction } & 1\end{array}$

2 Form factors of the order operator 3

2.1 Form factors of exponential fields in the sinh-Gordon model 5

$\begin{array}{lll}2.2 & \text { Construction of the order operator for the massless flow } & 7\end{array}$

3 Sinh-Gordon twist field and the disorder operator $\quad 14$

3.1 Semi-local form factors in the sinh-Gordon model 14

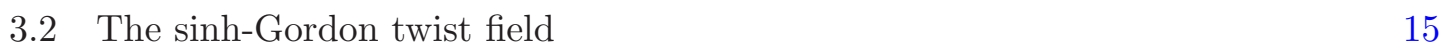

$\begin{array}{ll}3.3 \text { Construction of the disorder operator } & 16\end{array}$

4 Conclusions 18

$\begin{array}{ll}\text { A Form factor bootstrap for the massless flow } & 19\end{array}$

A.1 The recursion relations for the form factor polynomials 20

$\begin{array}{ll}\text { A.2 Solution for the order operator } & 21\end{array}$

A.3 Solution for the disorder operator 22

B Form factor bootstrap for semi-local fields $\quad 22$

C Limits of 5 and 6 particle form factor polynomials 24

\section{Introduction}

The staircase flow has attracted considerable interest since Al.B. Zamolodchikov's intriguing preprint (later published as [1]) appeared more than 20 years ago. In his work, Zamolodchikov observed that the S-matrix of the sinh-Gordon quantum field theory (QFT) in two dimensions can be analytically continued away from the self-dual point of the model to complex values of the coupling in such a way that the resulting S-matrix makes perfect sense as a scattering theory. Due to the lack of a proper Lagrangian description, he used the thermodynamic Bethe Ansatz (TBA) to study the properties of the analytically continued model. The $c$-function obtained from the TBA equations showed a peculiar behaviour as the real parameter $\theta_{0}$ encoding this continuation increased: a 'staircase' of more and more clearly defined plateaux appeared, at heights equal to the central charges $c=1-6 / p(p+1)$ of the conformal minimal models $\mathcal{M}_{p}$, and in the intervals between the plateaux the flow was found to approximate the crossovers $\mathcal{M}_{p} \rightarrow \mathcal{M}_{p-1}$ between the minimal models generated by the integrable perturbing operator $\phi_{1,3}$. This observation led to the interpretation of the model in this so-called roaming limit as a field theory describing a renormalisation 
group (RG) flow that passes by the successive conformal minimal models, eventually ending in a trivial IR fixed point which corresponds to a free massive Majorana fermion, i.e. the scaling Ising model. The larger the parameter $\theta_{0}$, the closer the flow approaches each minimal model fixed point and the longer time it spends in its vicinity, where the roaming model can be described as a combined perturbation by the relevant and irrelevant fields $\phi_{1,3}$ and $\phi_{3,1}[1,2]$. Subsequent work resulted in a variety of generalisations of Zamolodchikov's construction, giving evidence for further families of RG trajectories interpolating between other sequences of RG fixed points [3-6].

A different approach was taken in [7], in which the authors addressed the calculation of the $c$-function defined by the $c$-theorem $[8,9]$ using a spectral series in terms of the form factors of the trace of the stress-energy tensor $\Theta[10,11]$. The resulting $c$-function, although not fully identical with the TBA $c$-function, displays the same plateaux corresponding to the central charges of the series of conformal minimal models. The central observation of this work was that the support of the form factor integrals in the spectral sum comes from specific regions in the multi-dimensional rapidity space, a phenomenon dubbed relocalisation. These regions are essentially hypercubes of size $\mathcal{O}(1)$ with centre coordinates in rapidity space that are integer or half-integer multiples of the roaming parameter $\theta_{0}$. Since $\theta_{0}$ is sent to infinity in the roaming limit, such hypercubes eventually grow infinitely separated and become independent cells, from which the form factors of the trace of stress-energy tensor $\Theta$ in the perturbed minimal models can be reconstructed.

A logical next step is to attempt the construction of the form factors of other operators besides $\Theta$ from the roaming trajectories. For step $k=1$ corresponding to the thermal perturbation of the critical Ising model, the form factors of the sinh-Gordon field $\phi$ have been observed to reconstruct the form factors of the magnetisation operator merely by sending $\theta_{0} \rightarrow \infty$ [12]. For other steps, however, the limiting procedure is much more difficult, as the relocalisation patterns studied in [7] come to play a crucial role in the behaviour of the continued sinh-Gordon form factors.

The step $k=2$ in the roaming limit, corresponding to the massless flow from the tricritical to the critical Ising model, is particularly interesting, since besides the presence of the relocalisation patterns, the form factors of $\Theta \sim \phi_{1,3}$ were explicitly constructed in the earlier work [13], together with the form factors of the order operator $\Phi$ and the disorder operator $\tilde{\Phi}$. It was verified in [7] that the proposed roaming limit for the form factors of the particular sinh-Gordon operator $\Theta$ indeed matches the results in [13]. Motivated by the known analytic expressions for the form factors of $\Phi$ and $\tilde{\Phi}$ in the flow between the tricritical and critical Ising models, in this work we take the first steps towards the construction of the form factors of other operators besides $\Theta$ in a non trivial case by showing that they can be obtained as roaming limits of sinh-Gordon form factors. This leads to a better understanding of the roaming limits, especially with regard to the role played by CPT symmetry. In addition, to obtain form factors of $\tilde{\Phi}$ it is necessary to construct a new solution of the sinh-Gordon form factor bootstrap which corresponds to a semi-local operator, a.k.a. a twist field.

Our paper is organised as follows. In section 2 we briefly discuss some general properties of form factors and their generalisation to massless theories. We show that the form factors of the order operator $\Phi$ can indeed be obtained from the form factors of the odd 
scaling field : $\sinh \frac{g}{2} \phi:$ This construction necessitates a CPT symmetrisation step, which is argued to be a general ingredient of the roaming limit procedure. We also show that similarly to the Ising model, the order operator can be obtaned from the form factors of the elementary sinh-Gordon field as well. Section 3 is devoted to the disorder operator $\tilde{\Phi}$. Due its semi-local nature, it is first necessary to solve the sinh-Gordon form factor bootstrap for semi-local operators; we construct the minimal solution of the recursion up to 6 particles. Using the $\Delta$-theorem sum rule [14] it is shown that the field defined by the minimal solution is just the off-critical version of the twist operator known from the free massless scalar field theory. We then show that in the roaming limit the form factors of the twist field reproduce those of the disorder operator $\tilde{\Phi}$. Our conclusions are presented in section 4. To keep the main line of argument clear, some of the more technical details of the calculations are relegated to appendices.

\section{Form factors of the order operator}

In the following, we discuss two-dimensional relativistically invariant quantum field theories. Form factors are matrix elements of (semi-)local operators $O(x, t)$ between the vacuum and asymptotic states, i.e.,

$$
F_{\alpha_{1}, \ldots, \alpha_{n}}^{O}\left(\theta_{1}, \ldots, \theta_{n}\right)=\left\langle 0|O(0,0)| \theta_{1}, \ldots \theta_{n}\right\rangle_{\alpha_{1}, \ldots, \alpha_{n}} .
$$

In massive theories, the asymptotic states correspond to multi-particle excitations, with dispersion relation $(E, p)=\left(m_{\alpha_{i}} \cosh \theta, m_{\alpha_{i}} \sinh \theta\right)$, where $\alpha_{i}$ indicates the particle species. In such models, any multi-particle state can be constructed from vacuum state by means of the particle creation operators $A_{\alpha_{i}}^{\dagger}(\theta)$ by

$$
\left|\theta_{1}, \theta_{2}, \ldots, \theta_{n}\right\rangle_{\alpha_{1}, \ldots, \alpha_{n}}=A_{\alpha_{1}}^{\dagger}\left(\theta_{1}\right) A_{\alpha_{2}}^{\dagger}\left(\theta_{2}\right) \ldots A_{n}^{\dagger}\left(\theta_{n}\right)|0\rangle
$$

where the operator $A_{\alpha_{i}}^{\dagger}(\theta)$ creates a particle of species $\alpha_{i}$ with rapidity $\theta$ and $|0\rangle$ is the vacuum state of the theory. In an integrable QFT with factorised scattering, the creation and annihilation operators $A_{\alpha_{i}}^{\dagger}(\theta)$ and $A_{\alpha_{i}}(\theta)$ satisfy the Zamolodchikov-Faddeev (ZF) algebra

$$
\begin{aligned}
& A_{\alpha_{i}}^{\dagger}\left(\theta_{i}\right) A_{\alpha_{j}}^{\dagger}\left(\theta_{j}\right)=S_{\alpha_{i}, \alpha_{j}}\left(\theta_{i}-\theta_{j}\right) A_{\alpha_{j}}^{\dagger}\left(\theta_{j}\right) A_{\alpha_{i}}^{\dagger}\left(\theta_{i}\right), \\
& A_{\alpha_{i}}\left(\theta_{i}\right) A_{\alpha_{j}}\left(\theta_{j}\right)=S_{\alpha_{i}, \alpha_{j}}\left(\theta_{i}-\theta_{j}\right) A_{\alpha_{i}}\left(\theta_{j}\right) A_{\alpha_{i}}\left(\theta_{i}\right), \\
& A_{\alpha_{i}}\left(\theta_{i}\right) A_{\alpha_{j}}^{\dagger}\left(\theta_{j}\right)=S_{\alpha_{i}, \alpha_{j}}\left(\theta_{j}-\theta_{i}\right) A_{\alpha_{j}}^{\dagger}\left(\theta_{j}\right) A_{\alpha_{i}}\left(\theta_{i}\right)+\delta_{\alpha_{i}, \alpha_{j}} 2 \pi \delta\left(\theta_{1}-\theta_{2}\right) \mathbf{1},
\end{aligned}
$$

where $S_{\alpha_{i}, \alpha_{j}}\left(\theta_{i}-\theta_{j}\right)$ are the two-particle S-matrices of the theory. However, (2.1) can be applied to massless theories as well using the concepts of massless scattering theory $[15,16]$ with the particle species labels including also a distinction between left-movers $(L)$ and right-movers $(R)$.

The essence of the massless S-matrix approach can be formulated by taking an appropriate limit of a massive integrable model; for simplicity we restrict ourselves here to the case of a single self-conjugate particle since this the case we are mostly interested in. In 
the massless model, we have right- and left-moving particles, whose creation operators can be obtained from the massive creation operators as

$$
A_{R, L}^{\dagger}(\theta)=\lim _{\theta_{0} \rightarrow \infty} A^{\dagger}\left(\theta \pm \beta_{0} / 2\right),
$$

where the mass $m$ in the massive model must be sent to zero so that the scale $M=m e^{\beta_{0} / 2}$ stays finite; $M$ is eventually the cross-over scale along the resulting massless flow. The spectrum of the right-moving and left-moving particles is then given by

$$
\begin{aligned}
& R: p^{0}=p^{1}=\frac{M}{2} e^{\theta} \\
& L: p^{0}=-p^{1}=\frac{M}{2} e^{-\theta} .
\end{aligned}
$$

With the massless creation and annihilation operators the asymptotic states can be written similarly to the massive cases, and satisfy an algebra of the form (2.1) which can be obtained as the limit of the massive ZF algebra with S-matrices [13]

$$
\begin{aligned}
& S_{L L}(\theta)=S_{R R}(\theta)=S(\theta) \\
& S_{R L}(\theta)=\lim _{\beta_{0} \rightarrow \infty} S\left(\theta+\beta_{0}\right) \\
& S_{L R}(\theta)=\lim _{\beta_{0} \rightarrow \infty} S\left(-\theta-\beta_{0}\right) .
\end{aligned}
$$

In an integrable QFT the form factors of (semi-)local operators satisfy the so-called form factor bootstrap equations [17-19]

$$
\begin{aligned}
F_{\alpha_{1}, \ldots, \alpha_{i}, \alpha_{i+1}, \ldots \alpha_{n}}^{O}\left(\theta_{1}, \ldots \theta_{i}, \theta_{i+1}, \ldots, \theta_{n}\right)= & S_{\alpha_{i}, \alpha_{i+1}}\left(\theta_{i}-\theta_{i+1}\right) \\
& \times F_{\alpha_{1}, \ldots, \alpha_{i+1}, \alpha_{i}, \ldots \alpha_{n}}^{O}\left(\theta_{1}, \ldots \theta_{i+1}, \theta_{i}, \ldots, \theta_{n}\right) \\
F_{\alpha_{1}, \alpha_{2}, \ldots \alpha_{n}}^{O}\left(\theta_{1}+2 \pi i, \theta_{2}, \ldots, \theta_{n}\right)= & e^{2 \pi i \gamma} F_{\alpha_{2}, \ldots \alpha_{n}, \alpha_{1}}^{O}\left(\theta_{2}, \ldots, \theta_{n}, \theta_{1}\right) \\
-i \operatorname{Res}_{\theta^{\prime}=\theta+i \pi} F_{\alpha, \alpha, \alpha_{1}, \ldots \alpha_{n}}^{O}\left(\theta^{\prime}, \theta, \theta_{1}, \theta_{2}, \ldots, \theta_{n}\right)= & \left(1-e^{2 \pi i \gamma} \prod_{i=1}^{n} S_{\alpha, \alpha_{i}}\left(\theta-\theta_{i}\right)\right) \\
& \times F_{\alpha_{1}, \ldots \alpha_{n}}^{O}\left(\theta_{1}, \theta_{2}, \ldots, \theta_{n}\right) .
\end{aligned}
$$

The $e^{2 \pi i \gamma}$ factor in (2.5) and (2.6) is called the semi-local or mutual locality index of the operator $O$ with respect to the interpolating field $\phi$ and is defined via the condition

$$
O(x, t) \phi\left(y, t^{\prime}\right)=e^{2 \pi i \gamma} \phi\left(y, t^{\prime}\right) O(x, t)
$$

for space-like separated space-time points. Local operators correspond to $e^{2 \pi i \gamma}=1$, while fields with $e^{2 \pi i \gamma} \neq 1$ are called semi-local.

In addition, relativistic invariance implies

$$
F_{\alpha_{1}, \ldots, \alpha_{n}}^{O}\left(\theta_{1}+\Lambda, \ldots, \theta_{n}+\Lambda\right)=e^{s \Lambda} F_{\alpha_{1}, \ldots, \alpha_{n}}^{O}\left(\theta_{1}, \ldots, \theta_{n}\right),
$$

where $s$ is the Lorentz spin of the operator. As the models considered in this paper have no bound states, (2.4)-(2.6) and (2.7) give all the constraints for form factors of general (semi-)local operators. 


\subsection{Form factors of exponential fields in the sinh-Gordon model}

The sinh-Gordon model is defined by the Hamiltonian

$$
\begin{aligned}
H & =\int d x\left[\frac{1}{2} \pi^{2}+\frac{1}{2}\left(\partial_{x} \phi\right)^{2}+\frac{m_{0}^{2}}{g^{2}}: \cosh g \phi:\right], \\
{[\phi(t, x), \pi(t, y)] } & =i \delta(x-y)
\end{aligned}
$$

where $m_{0}$ is the classical particle mass and $g$ the coupling constant. The spectrum of the model consists of multi-particle states of a single massive bosonic particle with exact mass $m$. The two-particle $S$-matrix is [20]

$$
S(\theta)=\frac{\sinh (\theta)-\left(\omega-\omega^{-1}\right) / 2}{\sinh (\theta)+\left(\omega-\omega^{-1}\right) / 2}
$$

where $\theta=\theta_{1}-\theta_{2}$ is the relative rapidity of the particles,

$$
\omega=e^{i \frac{\pi B}{2}}
$$

and $B$ is related to the coupling $g$ by

$$
B=\frac{2 g^{2}}{8 \pi+g^{2}}
$$

For the sinh-Gordon model, solutions of the system (2.4)-(2.7) were first constructed in [11]. In [21], an important class of form factor solutions related to the exponential operators

$$
: e^{\kappa g \Phi}:
$$

was obtained, where $\kappa \in \mathbb{R}$. The exact vacuum expectation values of these operators were found in $[22]$ and are denoted here as

$$
G_{\kappa}=H_{0}^{\kappa}=\left\langle 0\left|: e^{\kappa g \Phi}:\right| 0\right\rangle .
$$

Introducing the notation

$$
O^{\kappa}=G_{\kappa}^{-1}: e^{\kappa g \Phi}:
$$

the form factors of normalised exponential operators take the following form [21]

$$
F_{n}^{\kappa}\left(\theta_{1}, \ldots, \theta_{n}\right)=\left\langle 0\left|O^{\kappa}\right| \theta_{1}, \ldots, \theta_{n}\right\rangle=\left(\frac{4 \sin \frac{\pi B}{2}}{\mathcal{N}}\right)^{n / 2} \frac{Q_{n}^{\kappa}\left(x_{1}, \ldots, x_{n}\right)}{\prod_{i<j}\left(x_{i}+x_{j}\right)} \prod_{i<j} f_{\min }\left(\theta_{i}-\theta_{j}\right)
$$

where $x_{i}=e^{\theta_{i}}$. In fact, apart from the overall normalisation, (2.15) gives the most general parametrisation for the form factors of local operators $[10,11]$. The minimal form factor $f_{\min }$ is

$$
\begin{aligned}
& f_{\min }(\theta, B)=\mathcal{N} \tilde{f}_{\min }(\theta, B) \\
& \tilde{f}_{\min }(\theta, B)=\exp \left[8 \int_{0}^{\infty} \frac{\mathrm{d} t}{t} \frac{\sinh \frac{B t}{4} \sinh \frac{t}{2}\left(1-\frac{B}{2}\right) \sinh \frac{t}{2}}{\sinh ^{2} t} \sin ^{2}\left[\frac{t(i \pi-\theta)}{2 \pi}\right]\right]
\end{aligned}
$$


which is a complex valued meromorphic function without singularities for real rapidities. This function tends to unity for large rapidities, i.e., $\lim _{\theta \rightarrow \pm \infty} f_{\min }(\theta)=1$, so long as the normalisation is chosen to be

$$
\mathcal{N}=f_{\min }(i \pi, B)=\exp \left[-4 \int_{0}^{\infty} \frac{\mathrm{d} t}{t} \frac{\sinh \frac{B t}{4} \sinh \frac{t}{2}\left(1-\frac{B}{2}\right) \sinh \frac{t}{2}}{\sinh ^{2} t}\right] .
$$

The functions $Q_{n}$ appearing in (2.15) are entire functions and completely symmetric in the variables $x_{i}$; for an operator with a power-like short distance singularity they can only grow exponentially for large values of the rapidities and are therefore restricted to be polynomials [23]. From now on we call these functions form factor polynomials; their growth at infinity is related to the ultraviolet scaling dimension of the operator, and solutions with the lowest possible growth at infinity, called minimal solutions, correspond to operators with the lowest possible conformal dimensions. Since the form (2.15) of the form factor is completely general, the dependence on the specific operator is carried by the form factor polynomials $Q_{n}$.

For the exponential operators discussed here, the polynomial part can be written as

$$
Q_{n}^{\kappa}=\operatorname{det} M_{i j}(\kappa),
$$

where $M$ is an $(n-1) \times(n-1)$ matrix with elements

$$
M_{i j}(\kappa)=[\kappa+i-j] \sigma_{2 i-j}^{(n)},
$$

where

$$
[n]=\frac{\sin n \frac{\pi B}{2}}{\sin \frac{\pi B}{2}}
$$

and $\sigma_{i}^{(n)}$ denotes the $i$ th symmetric polynomial of $n$ variables $x_{1}, \ldots, x_{n}$ defined by the generating function

$$
\prod_{i=1}^{n}\left(x+x_{i}\right)=\sum_{k=0}^{n} x^{n-k} \sigma_{k}^{(n)}\left(x_{1}, \ldots, x_{n}\right) .
$$

The upper index $(n)$ will be omitted in cases where this causes no confusion.

As the exponential operators are spinless, the total degree of the polynomials (2.18) must be the same as that of the denominator in (2.15); since the total degree of $Q_{n}^{\kappa}$ is $n(n-1) / 2$ this is indeed satisfied. The partial degree of the polynomials is at most $n-1$, which ensures that $\lim _{\theta_{i} \rightarrow \infty} F_{n}^{\kappa}\left(\theta_{1}, \ldots, \theta_{n}\right)$ is bounded by a constant. A further important property of the form factors of exponential operators is clustering (also called asymptotic factorisation) [14]:

$$
\lim _{\Lambda \rightarrow \infty} F_{r+l}^{O}\left(\theta_{1}+\Lambda, \ldots, \theta_{r}+\Lambda, \theta_{r+1}, \ldots \theta_{r+l}\right)=\frac{1}{\langle O\rangle} F_{r}^{O}\left(\theta_{1}, \ldots, \theta_{r}\right) F_{l}^{O}\left(\theta_{r+1}, \ldots \theta_{r+l}\right) .
$$

When applied to form factors of normalised exponential operators this can be written as

$$
\lim _{\Lambda \rightarrow \infty} F_{r+l}^{\kappa}\left(\theta_{1}+\Lambda, \ldots, \theta_{r}+\Lambda, \theta_{r+1}, \ldots \theta_{r+l}\right)=F_{r}^{\kappa}\left(\theta_{1}, \ldots, \theta_{r}\right) F_{l}^{\kappa}\left(\theta_{r+1}, \ldots \theta_{r+l}\right) .
$$

Finally, we remark that the form factors of the elementary field $\phi$ in the sinh-Gordon model are proportional to $\left.\frac{d F_{n}^{\kappa}}{d \kappa}\right|_{\kappa=0}$; in particular, the polynomial part is just $Q_{n}^{0}=\operatorname{det} M_{i j}(0)$. The form factors of $\phi$ are only non-zero when $n$ is odd. 


\subsection{Construction of the order operator for the massless flow}

Similarly to the sinh-Gordon form factors (2.15), the form factors of the massless flow can be written as [13]

$$
\begin{aligned}
F_{r, l}\left(\theta_{1}, \ldots, \theta_{r}, \theta_{1}^{\prime}, \ldots, \theta_{l}^{\prime}\right)= & H_{r, l} Q_{r, l}\left(x_{1}, \ldots, x_{r}, y_{1} \ldots y_{l}\right) \prod_{1 \leqslant i<j \leqslant r} \frac{f_{R R}\left(\theta_{i}-\theta_{j}\right)}{x_{i}+x_{j}} \\
& \times \prod_{i=1 j=1}^{r} \prod_{R L}^{l} f_{i}\left(\theta_{i}-\theta_{j}^{\prime}\right) \prod_{1 \leqslant i<j \leqslant l} \frac{f_{L L}\left(\theta_{i}^{\prime}-\theta_{j}^{\prime}\right)}{y_{i}+y_{j}} .
\end{aligned}
$$

In the above formula we omitted the reference to the specific operator; indices $r$ and $l$ refer to the number of right/left-moving particles, respectively. $H_{r, l}$ is a suitably chosen normalisation constant, and the minimal two-particle form factors are

$$
f_{R R}(\theta)=f_{L L}(\theta)=\sinh (\theta / 2)
$$

and

$$
f_{R L}(\theta)=\exp \left(\frac{\theta}{4}-\int_{0}^{\infty} \frac{d t}{t} \frac{\sin ^{2}\left(\frac{i \pi-\theta}{2 \pi} t\right)}{\sinh (t) \cosh (t / 2)}\right) .
$$

The $Q_{r, l}$ are functions of the variables $x_{i}=e^{\theta_{i}}$ and $y_{i}=e^{-\theta_{i}^{\prime}}$; for local operators, $Q_{r, l}$ is an entire function symmetric separately in the variables $x_{i}$ and in $y_{j}$, while for semi-local operators $(\gamma=1 / 2)$, it contains also the factor

$$
\sqrt{\prod_{i=1}^{r} x_{i} \prod_{j=1}^{l} y_{j}}
$$

For the order and disorder operators $\Phi$ and $\tilde{\Phi}$ the functions $Q_{r, l}$ were calculated in [13] based on the form factor bootstrap, however, in our work we slightly modify the normalisations $H_{r, l}$ for the form factors as described in appendix A.

To construct the order operator, one needs to find a suitable candidate. Note that the order operator of the massless flow is odd and its operator product expansion with itself contains the trace of the energy-momentum tensor, which is the perturbing field. The most natural candidate for a starting point is a scaling field in the sinh-Gordon model with these properties, which is given by

$$
\tilde{O}^{1 / 2}=G_{1 / 2}^{-1}: \sinh \frac{1}{2} g \phi:
$$

Its form factors are identical with those of either $O^{1 / 2}$ or $-O^{-1 / 2}$ whenever the number of particles is odd, but vanish when their number is even. The normalization factor is chosen as in (2.14).

The roaming limit procedure consists of the following steps. First the couplingdependent parameter $B$ is continued as [1]

$$
B \rightarrow B\left(\theta_{0}\right):=1+i \frac{2}{\pi} \theta_{0} .
$$


In the form factors of the sinh-Gordon fields $\phi$ and $\Theta$, making this substitution and sending $\theta_{0} \rightarrow \pm \infty$ is sufficient to recover the form factors of operators $\sigma$ and $\epsilon$ for the $k=1$ step corresponding to the thermal perturbation of the critical Ising model, in which $\sigma$ is the magnetization operator and $\epsilon$ is the energy operator. For other steps, however, it is necessary to make a second step, to identify the relevant regions of rapidity space which contribute to spectral sums when taking $\theta_{0}$ to infinity. Following [7], we introduce the concept of cells which are labeled by a sequence of monotonically increasing integers $p_{1}<p_{2} \ldots<p_{l}$ as follows

$$
\mathcal{C}=[\underbrace{p_{l}, p_{l}, \ldots, p_{l}}_{r_{p_{l}}}, \underbrace{p_{l-1}, p_{l-1}, \ldots, p_{l-1}}_{r_{p_{l-1}}}, \ldots, \underbrace{p_{1}, p_{1}, \ldots, p_{1}}_{r_{p_{1}}}]
$$

where $r_{p_{1}} \ldots, r_{p_{l}}$ indicate the number of occurrences of each number $p_{i}$, or equivalently the length of each block of integers. Such a sequence specifies the appropriate limit of the form factor to be taken, as specified in [7] for the trace of the stress energy tensor $\Theta$. In [7] it was shown that the dominant contribution to the spectral sum comes from regions in the rapidity space that are hypercubes of width $\mathcal{O}(1)$ and whose centre coordinates can be written as a set of some integers multiplied with $\theta_{0} / 2$. In addition, the only contributions surviving in the roaming limit are those of these dominant regions. The cell notation (2.26) introduced above identifies each of these regions with their centre coordinates in units of $\theta_{0} / 2$. Denoting the $i^{\text {th }}$ element of a cell by $\mathcal{C}_{i}$, the roaming limit for $\Theta$ can be written as

$$
F_{\mathcal{C}}^{\Theta}\left(\theta_{1}, \ldots, \theta_{n}\right)=\lim _{\theta_{0} \rightarrow \infty} \mathcal{N} e^{-\alpha \theta_{0}} F_{n}^{\Theta}\left(\theta_{1}+\mathcal{C}_{1} \theta_{0} / 2, \ldots, \theta_{n}+\mathcal{C}_{n} \theta_{0} / 2, B\left( \pm \theta_{0}\right)\right)
$$

with $\alpha$ chosen so that the limit is finite and $\mathcal{N}$ introduced to ensure proper normalisation of the minimal model $\Theta$ operator. For the thermally-perturbed Ising model, the only relevant cell is $[0,0]$ corresponding to no rapidity shifts; for $k=2$, i.e., the massless flow from the tricritical to critical Ising, the only relevant cells are of the type

$$
[\underbrace{1,1 \ldots, 1}_{r} \underbrace{-1,-1, \ldots,-1}_{l}]
$$

with $r, l$ even, so the minimal model form factors of $\Theta$ can be written as

$$
F_{r, l}^{\Theta}=\lim _{\theta_{0} \rightarrow \infty} \mathcal{N} e^{-2 \theta_{0}} F_{r+l}^{\Theta}\left(\theta_{1}+\theta_{0} / 2, \ldots, \theta_{r}+\theta_{0} / 2, \theta_{r+1}-\theta_{0} / 2, \ldots, \theta_{r+l}-\theta_{0}, B\left( \pm \theta_{0}\right)\right)
$$

in terms of the sinh-Gordon form factors and the phase factor $\mathcal{N}$ accounts for the difference between the proper normalisation of the two-particle form factor. We see that the cell structure accounts for the structure of the massless form factor through the separation of right- and left-moving particles. For higher flows the procedure described in [7] also generates so-called "magnonic" degrees of freedom that correspond to the internal structure of the kink excitations in the massless scattering theory. We omit these details as they are not needed in the sequel.

For the operator $\Theta$ a natural identification of the dominant integration regions [7] is given by the $c$-theorem spectral sum, which can be formulated as $[8,9]$

$$
c(R)=c(\infty)+\frac{3}{2} \int_{R}^{\infty} \mathrm{d} R^{\prime}\left(R^{\prime}\right)^{3}\left\langle\Theta\left(R^{\prime}\right) \Theta(0)\right\rangle
$$


in which $R$ is a length-scale. For the massive sinh-Gordon theory with $c(\infty)=0$, using the form factor expansion of the correlation function $\left\langle\Theta\left(R^{\prime}\right) \Theta(0)\right\rangle$, (2.29) can be rewritten with the form factors of $\Theta$ as

$$
c(r)=3 \sum_{n=0}^{\infty} \int_{\mathbb{R}^{\ltimes}} \frac{\mathrm{d} \theta_{1} \ldots \mathrm{d} \theta_{n}}{(2 \pi)^{n} n !} \frac{6+6 r E_{n}+3 r^{2} E_{n}^{2}+r^{3} E_{n}^{3}}{2 E_{n}^{4}}\left|m^{-2} F^{\Theta}\left(\theta_{1}, \ldots \theta_{n}\right)\right| e^{-r E},
$$

where $m$ is the physical mass, $r=m R$ and $E_{n}=\sum_{i=1}^{n} \cosh \theta_{i}$. As shown in [7], it is possible to carry out the roaming limit within the $c$-theorem sum rule; we briefly summarise the relevant arguments here.

First, the overall asymptotic behaviour of the form factors of $\Theta$ was found to be $e^{N \theta_{0}}$, with $N$ denoting the number of blocks in the cells, as long as all the blocks consist of an even number of members and the difference between the members of neighbouring blocks is 2 . If these criteria are not fulfilled, the asymptotics is always given by $e^{\omega \theta_{0}}$ with $\omega<N$. The number of blocks $N$ and the members of the cells are constrained on one hand by the number of variables of the form factors, and on the other hand by the exponential factor and the energy denominator entering the $c$-theorem sum rule integrands. To see the $k^{\text {th }}$ step in the roaming limit, the distance $r$ must scale as $r=\tilde{r} e^{-(k-1) \frac{\theta_{0}}{2}}$, where $\tilde{r}$ is finite, but due to the exponential factor in $(2.30),\left|\mathcal{C}_{i}\right| \leq k-1$. The contribution of cells with $\left|\mathcal{C}_{i}\right| \geq k-1$ are suppressed by a double exponential way. In addition to this, to minimise the energy term in the denominator one finds that

$$
\min _{i}\left(\mathcal{C}_{i}\right)=-\max _{i}\left(\mathcal{C}_{i}\right)
$$

These considerations leads to the following pattern for the dominant cells

$$
\left[p_{l}, p_{l}, \ldots p_{1}, p_{1}\right]: l \geq k,-p_{1}=p_{l}=k-1, p_{k}-p_{k-1}=2 \text { or } 0
$$

for the $k^{\text {th }}$ step of the staircase; for $k=2$ this reproduces (2.27). This yields the limiting procedure (2.28) for $\Theta$ with the normalisation of the operator fixed by the $c$-theorem.

For sinh-Gordon operators with form factors of even particle number, the $c$-theorem could be replaced by the $\Delta$-theorem [14] to define the dominant integration cells and hence the eventual limit of the form factors. If it converges, the $\Delta$-theorem states that if at some length scale $R$ the theory can be described by a CFT, then the difference of the conformal weight of an operator $O$ and its conformal weight in the IR limit can be calculated as

$$
D(R)-\Delta^{I R}=-\frac{1}{4 \pi\langle O\rangle} \int_{x^{2}>R} \mathrm{~d}^{2} x\langle\Theta(x) O(0)\rangle .
$$

Similarly to the $c$-theorem, (2.32) has a spectral representation

$$
D(r)-\Delta^{I R}=-\frac{1}{2\langle O\rangle} \sum_{n=1}^{\infty} \int \frac{\mathrm{d} \theta_{1} \ldots \mathrm{d} \theta_{n}}{(2 \pi)^{n} n !} \frac{e^{-r E_{n}}\left(1+E_{n} r\right)}{E_{n}^{2}} m^{-2} F^{\Theta}\left(\theta_{1}, \ldots, \theta_{n}\right) F^{O}\left(\theta_{n}, \ldots, \theta_{1}\right) .
$$


However, operators $\Phi$ and $\tilde{O}^{1 / 2}$ have non vanishing form factors only when $r+l$ is odd, and for these the $\Delta$-theorem is eventually vacuous, since $\Theta$ has only even form factors and also $\langle\Phi\rangle=0$. But the form factor expansion of the two point correlation function

$$
\left\langle\tilde{O}^{1 / 2}(x) \tilde{O}^{1 / 2}(0)\right\rangle=\sum_{n=1}^{\infty} \int \frac{\mathrm{d} \theta_{1}}{2 \pi} \ldots \frac{\mathrm{d} \theta_{2 n+1}}{2 \pi} \frac{1}{(2 n+1) !}\left|F^{1 / 2}\left(\theta_{1}, \ldots \theta_{2 n+1}\right)\right|^{2} e^{-r E_{2 n+1}}
$$

can be used to find the dominant cells for the roaming limit of the form factors of $\Phi$. Note that due to the definition of $\tilde{O}^{1 / 2}$ only form factors of $O^{1 / 2}$ with odd number of particles appear in (2.34).

The only essential difference between the structures of (2.30) and (2.34) is the absence of the energy denominator in the latter, which means that the condition

$$
\min _{i}\left(\mathcal{C}_{i}\right)=-\max _{i}\left(\mathcal{C}_{i}\right)
$$

is relaxed. Following the power counting method used in [7], the asymptotic behaviour of the form factors of $O^{1 / 2}$ can be obtained as

$$
e^{\frac{1}{4} N_{\text {odd }} \theta_{0}+\theta_{0} / 2},
$$

where $N_{\text {odd }}$ is the number of blocks with an odd number of members. For $k=2$, the exponential part in (2.34) allows cells with $\mathcal{C}_{i}= \pm 1$ only, and therefore the dominant cells for correlation function of $\tilde{O}$ are again (2.27), but now $r+l$ must be odd and at least 1 .

Having identified the dominant cells for the spectral sum of the roaming two-point function for the $k=2$ step, we can finally perform the limit at the level of the form factors. Consider the following limit for odd $n=r+l$

$$
\lim _{\theta_{0} \rightarrow \infty} F_{n}^{1 / 2}\left(\theta_{1}+\theta_{0}, \ldots, \theta_{r}+\theta_{0}, \theta_{1}^{\prime}, \ldots, \theta_{l}^{\prime}, B\left( \pm \theta_{0}\right)\right)
$$

where we used Lorentz invariance to rearrange the rapidity shifts. Note that due to the asymptotic behaviour (2.35) it is necessary to renormalise the form factor by an appropriate power of $e^{\theta_{0}}$ in order to obtain finite quantities. However, this power is independent of the particle number, therefore it makes sense to take the limit of the form factors similarly to the case of $\Theta$.

Let us analyse the limit now in detail. The limit of the unnormalised minimal form factors $\tilde{f}_{\text {min }}$ in the sinh-Gordon model is

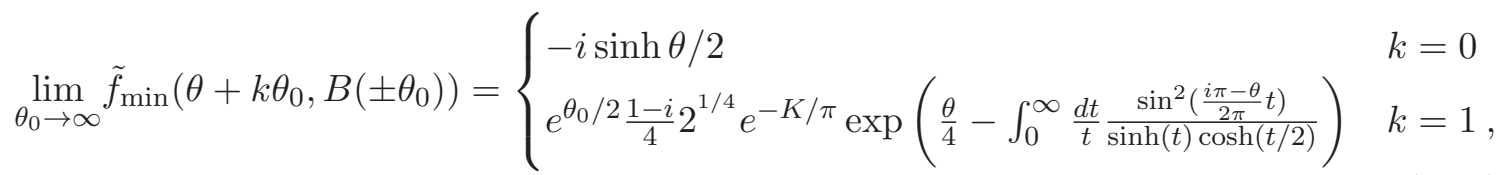

where $K$ is Catalan's constant. (2.37) reproduces the minimal form factors of the flow, $f_{R R}=f_{L L}=\sinh \theta / 2$ and (2.25) as obtained in [13], up to some constant normalization factor. For a sinh-Gordon form factor with $n$ rapidities, separated into $r$ right-moving and $l$ left-moving rapidities in the limiting procedure, the limit of the form factor and 
the minimal form factor normalizations including also the $(r l)^{\text {th }}$ power of the term $e^{\theta_{0} / 2}$ from $(2.37)$ in the leading order of $\mathcal{O}\left(e^{\theta_{0}}\right)$ is

$$
e^{r l \theta_{0} / 2} e^{\theta_{0}(n(4-n) / 4)} \cdot(-i)^{r(r-1) / 2+l(l-1) / 2}\left(\frac{1-i}{4} 2^{1 / 4} e^{-K / \pi}\right)^{l r} 2^{n(n-1) / 2} .
$$

For the limit of the kinematic pole denominator we obtain

$$
\prod_{1 \leq i<j \leq n}\left(x_{i}+x_{j}\right) \rightarrow e^{\theta_{0}(r(r-1) / 2+r l)} \prod_{1 \leq i<j \leq r}\left(x_{i}+x_{j}\right) \prod_{1 \leq n<m \leq l} \frac{y_{n}+y_{m}}{y_{n} y_{m}} \prod_{p=1}^{r} x_{p}^{l},
$$

where $x_{i}=e^{\theta_{i}}(i=1, \ldots, r)$ and $y_{i}=e^{-\theta_{j}^{\prime}}(j=1, \ldots, l)$. Note that in order to match the structure of the massless form factor (2.23), the factors

$$
\prod_{1 \leq n<m \leq l} y_{n} y_{m} \prod_{p=1}^{r} x_{p}^{-l}
$$

resulting from the limit of denominator must be combined with the limit of the $Q_{n}$ polynomials to give the functions $\tilde{Q}_{r, l}$, generally resulting in a rational function of symmetric polynomials.

For the roaming limit of the $Q_{n}$ polynomials we present some explicit results. For $n=1$ the limit is simply

$$
e^{3 \theta_{0} / 4}
$$

where the \pm corresponds to taking the roaming limit with either the $B\left(\theta_{0}\right)$ or the $B\left(-\theta_{0}\right)$ substitution.

For $n=3$, the $\tilde{Q}_{r, l}$ functions obtained from the roaming limit together with the various powers of $e^{\theta_{0}}$ extracted from the different parts of the form factor function are given in the table below:

\begin{tabular}{|c|c|c|c|c|c|}
\hline$n=3$ & $\tilde{Q}_{r, l}$ & Normalisation & $Q_{n}$ & Denominator & Overall scaling \\
\hline$r=0$ & $y_{1} y_{2} y_{3}$ & $e^{3 \theta_{0} / 4}$ & 1 & 1 & $e^{3 \theta_{0} / 4}$ \\
\hline$r=1$ & $\frac{1}{x_{1}} \pm i\left(y_{1}+y_{2}\right)$ & $e^{7 \theta_{0} / 4}$ & $e^{\theta_{0}}$ & $e^{-2 \theta_{0}}$ & $e^{3 \theta_{0} / 4}$ \\
\hline$r=2$ & $\frac{1}{y_{1}} \pm i\left(x_{1}+x_{2}\right.$ & $e^{7 \theta_{0} / 4}$ & $e^{2 \theta_{0}}$ & $e^{-3 \theta_{0}}$ & $e^{3 \theta_{0} / 4}$ \\
\hline$r=3$ & $x_{1} x_{2} x_{3}$ & $e^{3 \theta_{0} / 4}$ & $e^{3 \theta_{0}}$ & $e^{-3 \theta_{0}}$ & $e^{3 \theta_{0} / 4}$ \\
\hline
\end{tabular}

For $n=5$ the corresponding tables are presented in appendix C.

We see that the overall scaling of the form factors is indeed independent of the number of particles and is equal to

$$
e^{3 \theta_{0} / 4}
$$

which can be proven using the power counting approach of [7]. This allows a general renormalisation of the form factors that is independent of the number of particles to be applied before taking the roaming limit. We also include a finite renormalisation so that the roaming limits of the one-particle form factors satisfy $\langle 0|\Phi| \theta\rangle_{R / L}=1$. This is a natural normalisation since we expect $\langle 0|\Phi| \theta\rangle_{R}=\langle 0|\Phi| \theta\rangle_{L}$ due to parity invariance. 
Comparing the resulting form factor polynomials with the known exact expressions for the minimal model form factors [13] (cf. also appendix A) shows that the form factor polynomials obtained from the roaming limit contain an additional imaginary part which changes sign when switching between the two possible roaming limits corresponding to $B\left(+\theta_{0}\right)$ and $B\left(-\theta_{0}\right)$.

The solution of this discrepancy lies in the observation that both the sinh-Gordon model and the massless flow are CPT invariant, since they are described by Hermitian relativistic invariant actions. However, the naive roaming continuation breaks hermiticity, and CPT takes $B\left(+\theta_{0}\right)$ into $B\left(-\theta_{0}\right)$ and vice versa. On the other hand, both roaming continuations yield a solution for the form factor bootstrap for the massless form factor bootstrap since the sinh-Gordon $S$-matrix (2.9) is mapped into the massless S-matrix. As a result, any solution for the sinh-Gordon form factor bootstrap is taken to a solution for the form factor bootstrap of the massless flow. However, the form factor bootstrap is linear, and the correspondence between its solutions and local operators is not necessarily trivial.

As a result of the above considerations we propose a further CPT symmetrisation step before the roaming limit and to consider the expression

$$
\begin{aligned}
\lim _{\theta_{0} \rightarrow \infty} & \left(\frac{F_{n}^{1 / 2}\left(\theta_{1}+\theta_{0}, \ldots, \theta_{r}+\theta_{0}, \theta_{1}^{\prime}, \ldots, \theta_{l}^{\prime}, B\left(+\theta_{0}\right)\right)}{2 F_{1}^{1 / 2}\left(0, B\left(+\theta_{0}\right)\right)}\right. \\
& \left.+\frac{F_{n}^{1 / 2}\left(\theta_{1}+\theta_{0}, \ldots, \theta_{r}+\theta_{0}, \theta_{1}^{\prime}, \ldots, \theta_{l}^{\prime}, B\left(-\theta_{0}\right)\right)}{2 F_{1}^{1 / 2}\left(0, B\left(-\theta_{0}\right)\right)}\right)
\end{aligned}
$$

for odd $n$. This results in the following result for the CPT symmetrised $Q_{r, l}$ functions as obtained from the roaming limit, which is equal to the known exact expressions ([13], cf. also appendix A)

$$
\begin{array}{ll}
Q_{r, 0}=\rho_{r}^{(r-1) / 2} & \text { for odd } r \\
Q_{r, 1}=\frac{\rho_{r}^{r / 2-1}}{\lambda_{1}^{r / 2}} & \text { for even } r \\
Q_{r, 2}=\rho_{r}^{(r-3) / 2} \sum_{k=0}^{r} \rho_{k} \lambda_{2}^{(k-r+1) / 2} & \text { for odd } r \\
Q_{r, 3}=\frac{\rho_{r}^{r / 2-2}}{\lambda_{3}^{r / 2-1}} \sum_{k=0}^{\prime} \rho_{k} \lambda_{2}^{k / 2} & \text { for even } r
\end{array}
$$

where $\rho_{k}$ denotes the $k$ th symmetric polynomial of variables $x_{1}, \ldots, x_{r}, \lambda_{k}$ denotes the $k$ th symmetric polynomial of variables $y_{1}, \ldots, y_{l}$ and the primed sum means summation on even indices.

The remaining task is to check the normalisation factors ([13], cf. also appendix A)

$$
H_{r, l}=2^{r(r-1) / 2+l(l-1) / 2} \gamma^{-r l / 2} i^{(r l+r+l-1) / 2},
$$

where $\gamma=\sqrt{2} e^{2 K / \pi}$. It is not difficult to see that this is identical with (2.38) when the latter is rescaled with the appropriate power of $e^{\theta_{0}}$. Therefore, the final form of the limit 
that gives the normalised form factors of the order operator is

$$
\begin{aligned}
F_{r, l}^{\Phi}=\lim _{\theta_{0} \rightarrow \infty}( & \frac{F_{n}^{1 / 2}\left(\theta_{1}+\theta_{0}, \ldots, \theta_{r}+\theta_{0}, \theta_{1}^{\prime}, \ldots, \theta_{l}^{\prime}, B\left(+\theta_{0}\right)\right)}{2 F_{1}^{1 / 2}\left(B\left(+\theta_{0}\right)\right)} \\
& \left.+\frac{F_{n}^{1 / 2}\left(\theta_{1}+\theta_{0}, \ldots, \theta_{r}+\theta_{0}, \theta_{1}^{\prime}, \ldots, \theta_{l}^{\prime}, B\left(-\theta_{0}\right)\right)}{2 F_{1}^{1 / 2}\left(B\left(-\theta_{0}\right)\right)}\right)
\end{aligned}
$$

for $n=r+l$ odd. Note that since the solution for the sinh-Gordon form factors is known for any number of particles in a closed form, our result goes beyond the one obtained in the massless form factor bootstrap since it gives the form factors in a closed form for any number of particles instead of a recursive construction.

In the above derivation, the form factors of the order operator for the massless flow were reconstructed from the form factors of $\tilde{O}^{1 / 2}$, which is proportional to $: \sinh \frac{1}{2} g \phi:$. In addition, it is easy to show that for the first step of the staircase, the form factors of the order operator in the thermal perturbed Ising model can also be obtained from this field, as for odd $n$, following the construction presented in [12] that consists of simply sending $\theta_{0}$ to $\infty$, one has

$$
\lim _{\theta_{0} \rightarrow \infty} \frac{F_{n}^{1 / 2}\left(\theta_{1}, \ldots, \theta_{n}, B\left( \pm \theta_{0}\right)\right)}{F_{1}^{1 / 2}\left(B\left( \pm \theta_{0}\right)\right)}=i^{(n-1) / 2} \prod_{1 \leq i<j \leq n} \tanh \left(\frac{\theta_{i}-\theta_{j}}{2}\right) .
$$

In [12], the form factors of the order operator in the thermal perturbed Ising model were reconstructed from the form factors of the sinh-Gordon field $\phi$ as

$$
\lim _{\theta_{0} \rightarrow \infty} \frac{F_{n}^{\phi}\left(\theta_{1}, \ldots, \theta_{n}, B\left( \pm \theta_{0}\right)\right)}{F_{1}^{\phi}\left(B\left( \pm \theta_{0}\right)\right)}=i^{(n-1) / 2} \prod_{1 \leq i<j \leq n} \tanh \left(\frac{\theta_{i}-\theta_{j}}{2}\right),
$$

note, however, that $\phi$ is not a scaling field.

It turns out that this works for the case $k=2$ as well: the dominant cells for $F_{n}^{\phi}$ are identical to those of $F_{n}^{1 / 2}$ for odd $n$, and the roaming limits of the polynomials $Q_{n}^{0}$ are proportional to the real parts of the limits of $Q_{n}^{1 / 2}$. Although their scaling with $\theta_{0}$ is different, the difference in the scaling exponent is the same for any choice $n=r+l$ provided $n$ is odd. Therefore one can also write

$$
F_{r, l}^{\Phi}=\lim _{\theta_{0} \rightarrow \infty} \frac{F_{n}^{\phi}\left(\theta_{1}+\theta_{0}, \ldots, \theta_{r}+\theta_{0}, \theta_{1}^{\prime}, \ldots, \theta_{l}^{\prime}, B\left( \pm \theta_{0}\right)\right)}{F_{1}^{\phi}\left(B\left( \pm \theta_{0}\right)\right)} .
$$

For $\phi$ there is no need of CPT symmetrisation; note that the roaming limit for the trace of the stress-energy tensor (2.28), together with the limit for the step $k=1$ order operator and for (2.46) is independent of the sign choice in $B\left( \pm \theta_{0}\right)$, therefore the CPT symmetrisation prescription can be trivially extended to cover that case as well. The fact that the same operator can be obtained as the roaming limit of more than one sinh-Gordon field is not surprising. In fact, many more solutions to the form factor equations of the massless flow can be obtained by taking limits of other sinh-Gordon fields. However, identifying them with concrete operators in the perturbed minimal model is far from trivial. For the case of the order field the existence of the known exact solution was helpful, since unfortunately in this case the $\Delta$-theorem sum rule (2.33) is vacuous. 


\section{Sinh-Gordon twist field and the disorder operator}

\subsection{Semi-local form factors in the sinh-Gordon model}

In contrast to the order field $\Phi$, the disorder field $\tilde{\Phi}$ is a semi-local operator with mutual locality index $e^{2 \pi i \gamma}=-1$ [13]. Therefore it is necessary to construct the form factors of some semi-local operator at the sinh-Gordon level, since the roaming limit cannot change the mutual locality index.

The form factor equations for a semi-local field in the sinh-Gordon model that are to be satisfied by the semi-local sinh-Gordon form factors are the following

$$
\begin{aligned}
F_{n}^{O}\left(\theta_{1}, \ldots \theta_{i}, \theta_{i+1}, \ldots, \theta_{n}\right) & =S\left(\theta_{i}-\theta_{i+1}\right) F_{n}^{O}\left(\theta_{1}, \ldots \theta_{i+1}, \theta_{i}, \ldots, \theta_{n}\right) \\
F_{n}^{O}\left(\theta_{1}+2 \pi i, \theta_{2}, \ldots, \theta_{n}\right) & =-F_{n}^{O}\left(\theta_{2}, \ldots, \theta_{n}, \theta_{1}\right) \\
-i \operatorname{Res}_{\theta^{\prime}=\theta+i \pi} F_{n}^{O}\left(\theta^{\prime}, \theta, \theta_{1}, \theta_{2}, \ldots, \theta_{n}\right) & =\left(1+\prod_{i=1}^{n} S\left(\theta-\theta_{i}\right)\right) F_{n}^{O}\left(\theta_{1}, \theta_{2}, \ldots, \theta_{n}\right),
\end{aligned}
$$

and the general solution can be written as

$$
F_{n}\left(\theta_{1}, \ldots, \theta_{n}\right)=H_{n}\left(\prod_{i=1}^{n} \sqrt{x_{i}}\right) Q_{n}\left(x_{1}, \ldots, x_{n}\right) \prod_{1 \leqslant i<j \leqslant n} \frac{f_{\min }\left(\theta_{i}-\theta_{j}\right)}{x_{i}+x_{j}},
$$

where the parametrisation ensures that $Q_{n}$ is a symmetric polynomial of the variables $\left\{x_{i}\right\}$ and that (3.1) and (3.2) are automatically satisfied as long as choosing the Riemann sheets of the square root function according to the prescriptions $\sqrt{e^{2 \pi i}}=-1$ and $\sqrt{e^{\pi i}}=+i^{1}$ From (3.4) and the Lorentz transformation property (2.7) it follows that for a semi-local operator with integer Lorentz spin $s$ all matrix elements containing an odd number of particles must vanish. Substituting (3.4) into (3.3) and fixing the normalisation as

$$
H_{n+2}=H_{n} \mu^{2}
$$

with

$$
\mu=\sqrt{\frac{4 \sin \frac{\pi B}{2}}{f_{\min }(i \pi)}},
$$

a recursion equation can be written for $Q_{n}$

$$
Q_{n+2}\left(-x, x, x_{1}, \ldots, x_{n}\right)=C_{n}\left(x, x_{1}, \ldots, x_{n}\right) Q_{n}\left(x_{1}, \ldots, x_{n}\right)
$$

with the kernel:

$$
C_{n}\left(x, x_{1}, \ldots, x_{n}\right)=\sum_{k=0}^{n} \sum_{m=0}^{k} x^{2(n-k)+m} \sigma_{k}^{(n)} \sigma_{k-m}^{(n)}(-1)^{k+1}[m]_{c},
$$

\footnotetext{
${ }^{1}$ It is also possible to switch to the other Riemann sheet by redefining the form factor with appropriate signs.
} 
where $\sum^{\prime}$ means summation for even indices only and we introduced the notation

$$
[m]_{c}=\left\{\begin{array}{ll}
\frac{\cos \frac{\pi B}{2} m}{\sin \frac{\pi B}{2}} & \text { for } m \in \mathbb{Z} \backslash\{0\} \\
\frac{1}{2 \sin \frac{\pi B}{2}} & \text { for } m=0
\end{array} .\right.
$$

Some details about the derivation of the above recursion are given in appendix B.

\subsection{The sinh-Gordon twist field}

Similarly to the case of local operators, if a given $Q_{n}$ is a solution of the recursion, then $Q_{n}^{\prime}=\sigma_{n-1}^{(n)} \sigma_{1}^{(n)} Q_{n}$ is also a solution (corresponding to $\partial \bar{\partial} O$ ). Therefore we focus on the so-called irreducible operators whose form factors cannot be factorised. For irreducible semi-local operators with Lorentz spin $s$ equal to zero, it follows from (3.4) that $Q_{0}$ and $Q_{2}$ must be a constant. In addition, a simple power counting shows that the minimal solution $Q_{n}$ must have a partial degree $n-2$ in each variable, since $C_{n}$ has a partial degree 2 . The total degree can be determined from the Lorentz spin zero condition with the result

$$
\begin{aligned}
\operatorname{Deg}_{\text {tot }}\left[Q_{n}\right] & =\frac{n^{2}}{2}-n \\
\operatorname{Deg}_{\text {part }}\left[Q_{n}\right] & =n-2 \text { for } n \geq 2 .
\end{aligned}
$$

Setting $Q_{0}=1$, it follows immediately that $Q_{2}=-[0]_{c}$. For $Q_{4}$, the most general symmetric polynomial of four variables that satisfies irreducibility and (3.10) is $A_{1}^{(4)} \sigma_{4}+A_{2}^{(4)} \sigma_{1} \sigma_{3}^{(4)}+$ $A_{3}^{(4)} \sigma_{2} \sigma_{2}$. Substituting this Ansatz into (3.8), the unknown coefficients $A_{k}^{(4)}$ can be determined. The solution for the coefficients turns out to be unique:

$$
Q_{4}\left(x_{1}, x_{2}, x_{3}, x_{4}\right)=[0]_{c}\left([0]_{c}\left(\sigma_{1} \sigma_{3}+\sigma_{2} \sigma_{2}\right)-\left([2]_{c}+2[0]_{c}\right) \sigma_{4}\right) .
$$

$Q_{6}$ can be determined in a similar way with the result

$$
\begin{aligned}
Q_{6}\left(x_{1}, \ldots, x_{6}\right)= & {[0]_{c}\left(-[0]_{c}^{2} \sigma_{2} \sigma_{2} \sigma_{3} \sigma_{5}-[0]_{c}^{2} \sigma_{2} \sigma_{2} \sigma_{4} \sigma_{4}+[1]_{c}^{2} \sigma_{2} \sigma_{2} \sigma_{2} \sigma_{6}\right.} \\
& -[0]_{c}^{2} \sigma_{1} \sigma_{3} \sigma_{4} \sigma_{4}+[1]_{c}^{2} \sigma_{4} \sigma_{4} \sigma_{4}-[0]_{c}^{2} \sigma_{1} \sigma_{3} \sigma_{3} \sigma_{5}+[0]_{c}^{2} \sigma_{3} \sigma_{4} \sigma_{5} \\
& +[0]_{c}\left([2]_{c}+[0]_{c}\right) \sigma_{1} \sigma_{2} \sigma_{4} \sigma_{5}+[0]_{c}^{2} \sigma_{2} \sigma_{5} \sigma_{5}+[0]_{c}^{2} \sigma_{1} \sigma_{2} \sigma_{3} \sigma_{6} \\
& +[0]_{c}\left([2]_{c}+[0]_{c}\right) \sigma_{3} \sigma_{3} \sigma_{6}-[0]_{c}\left([4]_{c}+5[2]_{c}+6[0]_{c}\right) \sigma_{2} \sigma_{4} \sigma_{6} \\
& +[1]_{c}^{2} \sigma_{1} \sigma_{1} \sigma_{5} \sigma_{5}+[0]_{c}^{2} \sigma_{1} \sigma_{1} \sigma_{4} \sigma_{6} \\
& -[0]_{c}\left(2[4]_{c}+5[2]_{c}+7[0]_{c}\right) \sigma_{1} \sigma_{5} \sigma_{6} \\
& \left.+[0]_{c}\left([6]_{c}+3[4]_{c}+6[2]_{c}+7[0]_{c}\right) \sigma_{6} \sigma_{6}\right) .
\end{aligned}
$$

$Q_{8}$ has also been constructed and is again uniquely determined; we omit its formula due to its length. In fact, the solution is unique for any number of particles. The reason is that when determining $Q_{n}$ from $Q_{n-2}$, the ambiguity must be a symmetric polynomial $K_{n}$ satisfying

$$
K_{n}\left(-x, x, x_{3}, \ldots, x_{n}\right)=0
$$

However, any such polynomial can be written as

$$
K_{n}\left(x_{1}, \ldots, x_{n}\right)=L_{n}\left(x_{1}, \ldots, x_{n}\right) \prod_{i<j}\left(x_{i}+x_{j}\right)
$$


where $L_{n}$ is an arbitrary symmetric polynomial. The degrees of $K_{n}$ are

$$
\begin{aligned}
\operatorname{Deg}_{\text {tot }}\left[K_{n}\right] & \geq \frac{n(n-1)}{2} \\
\operatorname{Deg}_{\text {part }}\left[K_{n}\right] & \geq n-1 \text { for } n \geq 1,
\end{aligned}
$$

which is higher than (3.10), so no such ambiguity exists for the minimal solution. The uniqueness of the solution is in marked contrast to the case of local sinh-Gordon form factors where the dimension of the linear space spanned grows by one for every level of the recursion [21]. This can be understood considering that the minimal local solutions are the exponential operators whose space is spanned by the normal-ordered powers of the sinh-Gordon field $\phi$, so one indeed expects a countable infinity of independent solutions.

We denote the unique minimal spinless semi-local operator constructed above by $\tau$. It can be checked explicitly that the form factors of $\tau$ satisfy the cluster property $(2.21)$ and therefore they are expected to correspond to a scaling field [14].

The operator $\tau$ can be completely identified using the $\Delta$-theorem sum rule (2.33). Since the sinh-Gordon model has a massive spectrum, the IR limit gives zero conformal weight. Therefore (2.33) equals the conformal weight of the operator in the UV limiting theory, which is the massless free boson. Evaluating the integral numerically for $n=2,4$ and 6 and for various values of the coupling strength $B$ the following results were obtained:

\begin{tabular}{|c|c|c|c|}
\hline $\mathrm{B}$ & 0.1 & 0.5 & 0.9 \\
\hline 2 & 0.0638324 & 0.067049 & 0.0681442 \\
\hline $2+4$ & 0.0624367 & 0.0618555 & 0.0613877 \\
\hline $2+4+6$ & 0.0624976 & 0.0627215 & 0.0625972 \\
\hline
\end{tabular}

In the massless free bosonic theory, there exists an (up to normalisation) unique field which is a conformal primary and changes the boundary condition of the boson field from periodic to anti-periodic and vice versa. This twist field has conformal weight $\Delta=1 / 16$ [24] and our considerations show that the above solution can be identified with its off-critical version; the primary nature of the field corresponds to the minimality of the solution; non-minimal semi-local solutions of the bootstrap are expected to correspond to conformal descendants.

\subsection{Construction of the disorder operator}

We perform the roaming limit of the twist field the same way as discussed in section 2.3. The parametrisation for the form factors of $\tilde{\Phi}$ can be written as (2.23). The square root factors in the sinh-Gordon form factors of $\tau$ have the limits

$$
\prod_{i=1}^{n} \sqrt{x_{i}} \rightarrow e^{\theta_{0} r / 2} \prod_{i=1}^{r} \sqrt{x_{i}} \prod_{j=1}^{l} \sqrt{y_{j}^{-1}} .
$$

Due to the lack of a closed formula for the function $Q$ for the twist field form factors, we cannot determine the dominant cells directly. However, a direct generalisation of the cell 
structure for $\Theta$ and $\Phi$ works. We can use (2.27) for this case, with the restriction that $l+r$ is even. Therefore, the dominant cells to be considered are as follows:

2 particles : $[1,1] \quad[1,-1] \quad[-1,-1]$

4 particles : $[1,1,1,1] \quad[1,1,1,-1] \quad[1,1,-1,-1] \quad[1,-1,-1,-1] \quad[-1,-1,-1,-1]$

etc.

For 2 particles, the scaling of the form factor constituents in the roaming limit is as follows

\begin{tabular}{|c|c|c|c|c|c|c|}
\hline$n=2$ & $\tilde{Q}_{r, l}$ & Normalisation & $Q_{n}$ & Denominator & Square root & Overall scaling \\
\hline$r=0$ & -1 & $e^{\theta_{0}}$ & $e^{-\theta_{0}}$ & 1 & 1 & 1 \\
\hline$r=1$ & -1 & $e^{3 \theta_{0} / 2}$ & $e^{-\theta_{0}}$ & $e^{-\theta_{0}}$ & $e^{\theta_{0} / 2}$ & 1 \\
\hline$r=2$ & -1 & $e^{\theta_{0}}$ & $e^{-\theta_{0}}$ & $e^{-\theta_{0}}$ & $e^{\theta_{0}}$ & 1 \\
\hline
\end{tabular}

while for 4 particles one has

\begin{tabular}{|c|c|c|c|c|c|c|}
\hline$n=4$ & $\tilde{Q}_{r, l}$ & Normalisation & $Q_{n}$ & Denominator & Square root & Overall scaling \\
\hline$r=0$ & $y_{1}^{2} y_{2}^{2} y_{3}^{2} y_{4}^{2}$ & 1 & 1 & 1 & 1 & 1 \\
\hline$r=1$ & $\frac{y_{1} y_{2} y_{3}}{x_{1}^{2}}$ & $e^{3 \theta_{0} / 2}$ & $e^{\theta}$ & $e^{-3 \theta_{0}}$ & $e^{\theta_{0} / 2}$ & 1 \\
\hline$r=2$ & $\frac{1}{x_{1} x_{2}}+y_{1} y_{2}$ & $e^{2 \theta_{0}}$ & $e^{2 \theta_{0}}$ & $e^{-5 \theta_{0}}$ & $e^{\theta_{0}}$ & 1 \\
\hline$r=3$ & $\frac{1}{y_{1}}$ & $e^{3 \theta_{0} / 2}$ & $e^{3 \theta_{0}}$ & $e^{-6 \theta_{0}}$ & $e^{3 \theta_{0} / 2}$ & 1 \\
\hline$r=4$ & $x_{1} x_{2} x_{3} x_{4}$ & 1 & $e^{4 \theta}$ & $e^{-6 \theta_{0}}$ & $e^{2 \theta_{0}}$ & 1 \\
\hline
\end{tabular}

For the 6 particle case the results are presented in appendix $\mathrm{C}$.

The exact form factors of $\tilde{\Phi}$ resulting from the massless form factor bootstrap are given by ([13], cf. also appendix A)

$$
\begin{array}{ll}
Q_{r, 0}=\rho_{r}^{(r-1) / 2} & \text { for even } r \\
Q_{r, 1}=\frac{\rho_{r}^{r / 2-1}}{\lambda_{1}^{r / 2}} & \text { for odd } r \\
Q_{r, 2}=\rho_{r}^{(r-3) / 2} \sum_{k=0}^{\prime} \rho_{k} \lambda_{2}^{(k-r+1) / 2} & \text { for even } r \\
Q_{r, 3}=\frac{\rho_{r}^{r / 2-2}}{\lambda_{3}^{r / 2-1}} \sum_{k=0}^{r} \rho_{k} \lambda_{2}^{k / 2} & \text { for odd } r,
\end{array}
$$

in which the primed sum means summation on even indices and which are indeed equal to the corresponding $\tilde{Q}$ functions multiplied by the appropriate square root factors.

A more careful examination shows that for $n=2$ and 6 , there is a sign difference between the limit of the twist field $Q$ functions and the exact expression (3.17). This alternating sign is, however, cancelled by the normalisation factors. The normalization of the massless bootstrap solution reads

$$
H_{r, l}=2^{r(r-1) / 2+l(l-1) / 2} \gamma^{-r l / 2} i^{(r l+r+l) / 2} \quad \text { for } r, l \text { even }
$$




$$
H_{r, l}=\sqrt{2} 2^{r(r-1) / 2+l(l-1) / 2} \gamma^{-(r l+1) / 2} i^{(r l+r+l-3) / 2} \quad \text { for } r, l \text { odd },
$$

which corresponds to $F_{0,0}=1$ and $F_{1,1}=1$ in the IR limit $\theta \rightarrow 0$. It is important to stress that the recursion equation separates into two subsystems, one of which relates form factors of the form $F_{2 k, 2 l}$, while the other subsystem couples form factors of the form $F_{2 k+1,2 l+1}$. Due to this decoupling, it is possible and even necessary to apply a slightly different normalisation for the cases $r, l$ even/odd when performing the roaming limit of $\tau$. The limit of the prefactors for the form factors of the twist field are

$$
\begin{array}{ll}
2^{r(r-1) / 2+l(l-1) / 2} \gamma^{-r l / 2} i^{(r l+r+l) / 2} i^{-\left(n^{2}\right) / 2} & \text { for } r, l \text { even } \\
2^{r(r-1) / 2+l(l-1) / 2} \gamma^{-(r l+1) / 2} i^{(r l+r+l-3) / 2} i^{-\left(n^{2}-3\right) / 2} & \text { for } r, l \text { odd } .
\end{array}
$$

For even $n,(-i)^{\frac{n^{2}}{2}}$ is +1 if $n=0,4,8, \ldots$, and -1 if $n=2,6,10, \ldots$ This alternating sign cancels out the \pm 1 factors from the limit of the polynomial of the twist field, hence the properly normalised limit of the sinh-Gordon form factors is defined as

$$
F_{r, l}^{\tilde{\Phi}}=\lim _{\theta_{0} \rightarrow \infty} F_{n}^{\tau}\left(\theta_{1}+\theta_{0}, \ldots, \theta_{r}+\theta_{0}, \theta_{1}^{\prime}, \ldots, \theta_{l}^{\prime}, B\left( \pm \theta_{0}\right)\right) \text { for } r, l \text { even }
$$

and

$$
F_{r, l}^{\tilde{\Phi}}=i^{-3 / 2} \sqrt{2} \gamma^{-1 / 2} \lim _{\theta_{0} \rightarrow \infty} F_{n}^{\tau}\left(\theta_{1}+\theta_{0}, \ldots, \theta_{r}+\theta_{0}, \theta_{1}^{\prime}, \ldots, \theta_{l}^{\prime}, B\left( \pm \theta_{0}\right)\right) \text { for } r, l \text { odd } .
$$

Note that the roaming limit of the twist field is independent on the sign of $\theta_{0}$, therefore the CPT symmetrisation is trivial for the case of the disorder operator.

As a further test of the roaming limit construction, it is instructive to check if $\tau$ reproduces the disorder operator in step $k=1$, i.e., the perturbed Ising model. Indeed, with the first few solutions of $Q^{\tau}$ it is easy to check explicitly that

$$
\lim _{\theta_{0} \rightarrow \infty} F_{n}^{\tau}\left(\theta_{1}, \ldots, \theta_{n}, B\left( \pm \theta_{0}\right)\right)=i^{n / 2} \prod_{1 \leq i<j \leq n} \tanh \left(\frac{\theta_{i}-\theta_{j}}{2}\right),
$$

which are the form factors of the disorder operator in the perturbed Ising model with vacuum expectation value normalized to unity [26].

\section{Conclusions}

In this paper we have shown that it is possible to obtain the form factors of the order and disorder operators of the massless RG flow interpolating between the tricritical and the critical Ising model as a properly defined roaming limit of certain sinh-Gordon form factors. These results are a natural next step after the construction of the form factors of the trace of the stress-energy tensor in [7], which was based on Zamolodchikov's original staircase idea [1]. Our results demonstrate that the roaming construction of form factors can be extended to other operators along the massless flows generated by the roaming limit. We explicitly matched the resulting form factors with the previously known explicit solutions [13]. For the order operator it is important to note that the roaming limit 
explicitly constructs all the form factors for any number of particles, while previously it was only possible to obtain form factors recursively. In this construction we also found that in general, the roaming limit must incorporate a CPT symmetrisation. From our field theoretic arguments we expect that this lesson is general for any future extensions of the roaming form factor approach.

The construction of the disorder field necessitated the consideration of semi-local operators in the sinh-Gordon model. We found that the minimal solution was unique, which is consistent with the identification of the operator with the twist field changing the boundary conditions of the boson from periodic to anti-periodic and vice versa. This identification is strongly supported by the estimate of the scaling dimension obtained from the $\Delta$-theorem.

There are several interesting open directions. Focusing on the massless flow, it is possible to construct the roaming limit for other operators. In principle, by solving the form factor bootstrap for the minimal model flow along the lines of [13] at least the first few form factors of these operators can be constructed for the flow enabling a direct comparison with the limit of sinh-Gordon form factors, which can support the validity of the limiting procedure. For operators whose form factors are non-vanishing for even particle numbers, the $\Delta$-theorem can help to identify the operator. An even more interesting issue is to extend the construction of form factors of various operators for flows between higher minimal models corresponding to higher steps in Zamolodchikov's staircase. As a consequence of the non-diagonal scattering, for higher steps the solution of the FF bootstrap equation is highly non-trivial and is presently unknown. Therefore the construction of form factors with the roaming approach seems very promising, even if the magnonic structure of the limit [7] indicates a non-trivial relation to form factors being written in the standard RSOS kink basis $[25,27]$. Therefore the main issue to be solved is the proper treatment of the magnonic structure in the roaming limit. An even more ambitious goal is to extend the construction to other known staircase flows, and to connect the form factors of their local operators with the Dynkin formulation of the corresponding magnonic TBA systems [28], as suggested in [7].

\section{Acknowledgments}

This research was supported by the Momentum grant LP2012-50 of the Hungarian Academy of Sciences, by the STFC under grant number ST/L000407/1, and by the Marie Curie network GATIS (gatis.desy.eu) of the European Union's Seventh Framework Programme FP7/2007-2013/ under REA Grant Agreement No 317089. We are grateful to István Szécsényi for helpful discussions and collaboration on related matters.

\section{A Form factor bootstrap for the massless flow}

In the following we briefly review the main steps of the form factor bootstrap for the QFT associated with the RG flow between the two fixed points. These results were previously obtained in [13]; however, matching the form factors to the roaming limit requires changes in the normalisation conventions, so we briefly give the necessary details here. 


\section{A.1 The recursion relations for the form factor polynomials}

As discussed in the main text, the spectrum of the theory consists of right-moving and left-moving particles with dispersion $p^{0}=p^{1}=\frac{M}{2} e^{\theta} / p^{0}=-p^{1}=\frac{M}{2} e^{-\theta}$, whereas with the massless creation and annihilation operators the asymptotic in- and out-states can be written similarly to the massive cases. The massless S matrices [13] are

$$
\begin{aligned}
& S_{L L}(\theta)=S_{R R}(\theta)=-1 \\
& S_{R L}(\theta)=\tanh (\theta / 2-i \pi / 4) .
\end{aligned}
$$

The form factors of the theory associated with the RG flow are defined as

$$
F_{\alpha_{1}, \ldots, \alpha_{n}}^{O}\left(\theta_{1}, \ldots, \theta_{n}\right)=\left\langle 0|O(0,0)| A_{\alpha_{1}}\left(\theta_{1}\right) \ldots A_{\alpha_{n}}\left(\theta_{n}\right)\right\rangle
$$

and must satisfy the equations

$$
\begin{aligned}
F_{\alpha_{1}, \ldots, \alpha_{i}, \alpha_{i+1}, \ldots \alpha_{n}}^{O}\left(\theta_{1}, \ldots \theta_{i}, \theta_{i+1}, \ldots, \theta_{n}\right) & =S_{\alpha_{i}, \alpha_{i+1}}\left(\theta_{i}-\theta_{i+1}\right) F_{\alpha_{1}, \ldots, \alpha_{i+1}, \alpha_{i}, \ldots \alpha_{n}}^{O}\left(\theta_{1}, \ldots \theta_{i+1}, \theta_{i}, \ldots, \theta_{n}\right) \\
F_{\alpha_{1}, \alpha_{2}, \ldots \alpha_{n}}^{O}\left(\theta_{1}+2 \pi i, \theta_{2}, \ldots, \theta_{n}\right) & = \pm F_{\alpha_{2}, \ldots \alpha_{n}, \alpha_{1}}^{O}\left(\theta_{2}, \ldots, \theta_{n}, \theta_{1}\right) \\
-i \operatorname{Res}_{\theta^{\prime}=\theta+i \pi} F_{\alpha, \alpha, \alpha_{1}, \ldots \alpha_{n}}^{O}\left(\theta^{\prime}, \theta, \theta_{1}, \theta_{2}, \ldots, \theta_{n}\right) & =\left(1 \mp \prod_{i=1}^{n} S_{\alpha, \alpha_{i}}\left(\theta-\theta_{i}\right)\right) F_{\alpha_{1}, \ldots \alpha_{n}}^{O}\left(\theta_{1}, \theta_{2}, \ldots, \theta_{n}\right),
\end{aligned}
$$

where $\alpha$ and $\alpha_{i}$ denotes either a right-moving (R) or a left-moving $(\mathrm{L})$ particle. The choice of signs in the cyclic permutation and kinematic pole equations corresponds to local (upper choice) or semi-local (lower choice) operators, the latter being relevant for the disorder field. The form factors are parametrised as

$$
\begin{aligned}
F_{r, l}\left(\theta_{1}, \ldots, \theta_{r}, \theta_{1}^{\prime}, \ldots, \theta_{l}^{\prime}\right)= & H_{r, l} Q_{r, l}\left(x_{1}, \ldots, x_{r}, y_{1} \ldots y_{l}\right) \prod_{1 \leqslant i<j \leqslant r} \frac{f_{R R}\left(\theta_{i}-\theta_{j}\right)}{x_{i}+x_{j}} \\
& \times \prod_{i=1 j=1}^{r} \prod_{R L}^{l}\left(\theta_{i}-\theta_{j}^{\prime}\right) \prod_{1 \leqslant i<j \leqslant l} \frac{f_{L L}\left(\theta_{i}^{\prime}-\theta_{j}^{\prime}\right)}{y_{i}+y_{j}}
\end{aligned}
$$

where $r, l$ are the number of right/left movers, $H_{r, l}$ is a normalisation constant, $Q_{r, l}$ are meromorphic function of variables $x_{i}=e^{\theta_{i}}$ and $y_{i}=e^{-\theta_{i}^{\prime}}$ and the functions $f_{R R}, f_{L L}$ and $f_{R L}$ are the minimal form factors $f_{R R}(\theta)=f_{L L}(\theta)=\sinh (\theta / 2)$ and

$$
f_{R L}(\theta)=\exp \left(\frac{\theta}{4}-\int_{0}^{\infty} \frac{d t}{t} \frac{\sin ^{2}\left(\frac{i \pi-\theta}{2 \pi} t\right)}{\sinh (t) \cosh (t / 2)}\right),
$$

which solve

$$
f_{\alpha, \beta}(\theta)=S_{\alpha, \beta}(\theta) f_{\alpha, \beta}(\theta+2 \pi i) .
$$

In addition, function $f_{R L}(\theta)$ satisfies

$$
f_{R L}(\theta \pm i \pi) f_{R L}(\theta)=i \gamma\left(1 \pm i e^{-\theta}\right)^{-1}
$$

where $\gamma=\sqrt{2} e^{2 K / \pi}$ and $K$ is Catalan's constant. For the disorder operator the $Q_{r, l}$ functions contain a factor

$$
\sqrt{\prod_{i=1}^{r} x_{i} \prod_{j=1}^{l} y_{j}}
$$


The kinematical pole equation (A.4) gives a recurrence relation for the polynomials. With the normalisation

$$
H_{r+2, l}=i^{l+1} 2^{2 r+1} \gamma^{-l} H_{r, l},
$$

the right-mover recurrence relations for the polynomials read

$$
Q_{r+2, l}\left(-x, x, x_{1}, \ldots, y_{1}, \ldots\right)=i^{r-l+1} x^{r-l+1} \frac{\rho_{r}}{\lambda_{l}} \sum_{k=0}^{l}(-i x)^{k} \lambda_{k}\left(\left\{y_{j}\right\}\right) Q_{r, l},
$$

where $\rho_{r}$ denotes the $r$ th elementary symmetric polynomial of variables $x_{1}, \ldots, x_{r}$ and $\lambda_{l}$ denotes the $l$ th elementary symmetric polynomial of variables $y_{1}, \ldots, y_{l}$. For the leftmovers one obtains

$$
Q_{r, l+2}\left(, x \ldots, y_{1}, \ldots, y_{l}, y,-y\right)=(-i)^{l-r+1} y^{l-r+1} \frac{\lambda_{l}}{\rho_{r}} \sum_{k=0}^{r^{\prime}}(-i y)^{k} \rho_{k}\left(\left\{x_{i}\right\}\right) Q_{r, l}
$$

with the choice

$$
H_{r, l+2}=i^{r+1} 2^{2 l+1} \gamma^{-r} H_{r, l} .
$$

Equations (A.10) and (A.11) differ from the corresponding equations in [13] by some powers of $i$. As a consequence, the recursion relation for the normalisation constants is also slightly modified.

\section{A.2 Solution for the order operator}

For the order operator we require the normalisation $F_{1,0}=F_{0.1}=1$, or equivalently $Q_{1,0}=Q_{0.1}=1$. Then the solution for the $Q$ is [13]

$$
\begin{array}{rlr}
Q_{r, 0} & =\rho_{r}^{(r-1) / 2} & \text { for odd } r \\
Q_{r, 1}=\frac{\rho_{r}^{r / 2-1}}{\lambda_{1}^{r / 2}} & \text { for even } r \\
Q_{r, 2}=\rho_{r}^{(r-3) / 2} \sum_{k=0}^{r} \rho_{k} \lambda_{2}^{(k-r+1) / 2} & \text { for odd } r \\
Q_{r, 3}=\frac{\rho_{r}^{r / 2-2}}{\lambda_{3}^{r / 2-1}} \sum_{k=0}^{\prime} \rho_{k} \lambda_{2}^{k / 2} & \text { for even } r
\end{array}
$$

and the primed sum means summation on even indices. To obtain the functions $Q_{0, l}, Q_{1, l}$, $Q_{2, l}$ and $Q_{3, l}$ one simply needs to exchange the elementary symmetric polynomials $\rho \longleftrightarrow \lambda$ in (A.13). The explicit normalisation constants are

$$
H_{r, l}=2^{r(r-1) / 2+l(l-1) / 2} \gamma^{-r l / 2} i^{(r l+r+l-1) / 2} .
$$




\section{A.3 Solution for the disorder operator}

For the disorder operator $\widetilde{\Phi}$, we stipulate $F_{0.0}=1$ and also $F_{1,1}=1$ in the IR limit, i.e. when the rapidity difference in the form factor tends to 0 . From these constraints we have $Q_{0,0}=1$ and

$$
Q_{1,1}=\frac{1}{\rho_{1}^{1 / 2} \lambda_{1}^{1 / 2}},
$$

where the choice of $Q_{1,1}$ is made unique by requiring that the operator has a zero Lorentz spin and is symmetric under parity that swaps left-movers with right-movers. With these initial conditions, the $Q_{r, l}$ functions are the following [13]

$$
\begin{array}{ll}
Q_{r, 0}=\rho_{r}^{(r-1) / 2} & \text { for even } r \\
Q_{r, 1}=\frac{\rho_{r}^{r / 2-1}}{\lambda_{1}^{r / 2}} & \text { for odd } r \\
Q_{r, 2}=\rho_{r}^{(r-3) / 2} \sum_{k=0}^{r} \rho_{k} \lambda_{2}^{(k-r+1) / 2} & \text { for even } r \\
Q_{r, 3}=\frac{\rho_{r}^{r / 2-2}}{\lambda_{3}^{r / 2-1}} \sum_{k=0}^{r} \rho_{k} \lambda_{2}^{k / 2} & \text { for odd } r,
\end{array}
$$

in which the primed sum means summation on even indices and which contain the square root type products that are necessary to fulfil (A.3). These functions, again, are consistent with (A.10). Similarly to (A.13), for the functions $Q_{0, l}, Q_{1, l}, Q_{2, l}$ and $Q_{3, l}$ one simply needs to exchange the elementary symmetric polynomials $\rho \longleftrightarrow \lambda$ in (A.15).

The normalisation is chosen in a slightly different way for odd-odd and even-even form factors:

$$
\begin{array}{ll}
H_{r, l}=2^{r(r-1) / 2+l(l-1) / 2} \gamma^{-r l / 2} i^{(r l+r+l) / 2} & \text { for both } r, l \text { even } \\
H_{r, l}=\sqrt{2} 2^{r(r-1) / 2+l(l-1) / 2} \gamma^{-(r l+1) / 2} i^{(r l+r+l-3) / 2} & \text { for both } r, l \text { odd } .
\end{array}
$$

\section{B Form factor bootstrap for semi-local fields}

In view of the lack of bound state particles in the sinh-Gordon model, the starting equations for the bootstrap are

$$
\begin{aligned}
F_{n}^{O}\left(\theta_{1}, \ldots \theta_{i}, \theta_{i+1}, \ldots, \theta_{n}\right) & =S\left(\theta_{i}-\theta_{i+1}\right) F_{n}^{O}\left(\theta_{1}, \ldots \theta_{i+1}, \theta_{i}, \ldots, \theta_{n}\right) \\
F_{n}^{O}\left(\theta_{1}+2 \pi i, \theta_{2}, \ldots, \theta_{n}\right) & =-F_{n}^{O}\left(\theta_{2}, \ldots, \theta_{n}, \theta_{1}\right) \\
-i \operatorname{Res}_{\theta^{\prime}=\theta+i \pi} F_{n}^{O}\left(\theta^{\prime}, \theta, \theta_{1}, \theta_{2}, \ldots, \theta_{n}\right) & =\left(1+\prod_{i=1}^{n} S\left(\theta-\theta_{i}\right)\right) F_{n}^{O}\left(\theta_{1}, \theta_{2}, \ldots, \theta_{n}\right) .
\end{aligned}
$$

Hence the most general form of such form factors can be written

$$
F_{n}\left(\theta_{1}, \ldots, \theta_{n}\right)=H_{n}\left(\prod_{i=1}^{n} \sqrt{x_{i}}\right) Q_{n}\left(x_{1}, \ldots, x_{n}\right) \prod_{1 \leqslant i<j \leqslant n} \frac{f_{\min }\left(\theta_{i}-\theta_{j}\right)}{x_{i}+x_{j}},
$$


where $Q_{n}$ is a symmetric polynomial of the variables $\left\{x_{i}\right\}$; this satisfies equations (B.1)(B.2).

Plugging (B.4) into the kinematical singularity equation (B.3) gives a recurrence relation

$$
\begin{aligned}
& -H_{n+2} Q_{n+2}\left(-x, x, x_{1}, \ldots, x_{n}\right) f_{\min }(i \pi) \prod_{i=1}^{n} \frac{f_{\min }\left(\theta+i \pi-\theta_{i}\right) f_{\min }\left(\theta-\theta_{i}\right)}{x_{i}^{2}-x^{2}} \\
& =H_{n}\left(1+\prod_{i=1}^{n} S\left(\theta-\theta_{i}\right)\right) Q_{n}\left(x_{1}, \ldots, x_{n}\right) .
\end{aligned}
$$

Using the identity

$$
f_{\min }(\theta+i \pi) f_{\min }(\theta)=\frac{\sinh (\theta)}{\sinh (\theta)+\left(\omega-\omega^{-1}\right) / 2}
$$

and also

$$
\left(1+\prod_{i=1}^{n} S\left(\theta-\theta_{i}\right)\right)=\frac{\prod_{i=1}^{n} x^{2}-x_{i}^{2}+x x_{i}\left(\omega-\omega^{-1}\right)+\prod_{i=1}^{n} x^{2}-x_{i}^{2}-x x_{i}\left(\omega-\omega^{-1}\right)}{\prod_{i=1}^{n} x^{2}-x_{i}^{2}+x x_{i}\left(\omega-\omega^{-1}\right)},
$$

resulting from (2.9), the recursion can be written as

$$
\begin{aligned}
& -(-1)^{n} H_{n+2} f_{\min }(i \pi) Q_{n+2}\left(-x, x, x_{1}, \ldots, x_{n}\right)= \\
& {\left[\prod_{i=1}^{n} x^{2}-x_{i}^{2}+x x_{i}\left(\omega-\omega^{-1}\right)+\prod_{i=1}^{n} x^{2}-x_{i}^{2}-x x_{i}\left(\omega-\omega^{-1}\right)\right] H_{n} Q_{n} .}
\end{aligned}
$$

where $(-1)^{n}=1$ since $n$ is even. Introducing the quantity

$$
\mu=\sqrt{\frac{4 \sin \frac{\pi B}{2}}{f_{\min }(i \pi)}}
$$

we can define

$$
H_{n+2}=H_{n} \mu^{2}
$$

Then the recurrence relation takes the form

$Q_{n+2}\left(-x, x, x_{1}, \ldots, x_{n}\right)=\frac{-1}{4 \sin \frac{\pi B}{2}}\left[\prod_{i=1}^{n} x^{2}-x_{i}^{2}+x x_{i}\left(\omega-\omega^{-1}\right)+\prod_{i=1}^{n} x^{2}-x_{i}^{2}-x x_{i}\left(\omega-\omega^{-1}\right)\right] Q_{n}$,

which can be simplified to

$$
Q_{n+2}\left(-x, x, x_{1}, \ldots, x_{n}\right)=C_{n}\left(x, x_{1}, \ldots, x_{n}\right) Q_{n}\left(x_{1}, \ldots, x_{n}\right)
$$

where

$$
C_{n}\left(x, x_{1}, \ldots, x_{n}\right)=\frac{-1}{4 \sin \frac{\pi B}{2}}\left[\prod_{i=1}^{n}\left(x+\omega x_{i}\right)\left(x-\omega^{-1} x_{i}\right)+\prod_{i=1}^{n}\left(x-\omega x_{i}\right)\left(x+\omega^{-1} x_{i}\right)\right] .
$$


We proceed by manipulating $C_{n}$. Using the generator function of the elementary symmetric polynomials $\prod_{i=1}^{n}\left(x+\omega x_{i}\right)=\sum_{k=0}^{n} \omega^{k} x^{n-k} \sigma_{k}^{(n)}$ and $\prod_{i=1}^{n}\left(x-\omega^{-1} x_{i}\right)=\sum_{l=0}^{n} \omega^{-l}(-1)^{l} x^{n-l} \sigma_{l}^{(n)}$ and exploiting the fact that the two products in (B.10) differ only by $\omega \rightarrow-\omega$ yields

$$
C_{n}\left(x, x_{1}, \ldots, x_{n}\right)=\frac{-1}{4 \sin \frac{\pi B}{2}}\left[\sum_{k=0}^{n} \sum_{l=0}^{n} \omega^{k-l} x^{2 n-k-l} \sigma_{k}^{(n)} \sigma_{l}^{(n)}\left(1+(-1)^{k+l}\right)(-1)^{l}\right] .
$$

Introducing the functions $[m]_{c}$

$$
[m]_{c}= \begin{cases}\frac{\cos \frac{\pi B}{2} m}{\sin \frac{\pi B}{2}} & \text { for } m \in \mathbb{Z} \backslash\{0\} \\ \frac{1}{2 \sin \frac{\pi B}{2}} & \text { for } m=0\end{cases}
$$

and rearranging the sums our final form for the recursion kernel is

$$
C_{n}\left(x, x_{1}, \ldots, x_{n}\right)=\sum_{k=0}^{n} \sum_{m=0}^{k} x^{2(n-k)+m} \sigma_{k}^{(n)} \sigma_{k-m}^{(n)}(-1)^{k+1}[m]_{c} .
$$

\section{Limits of 5 and 6 particle form factor polynomials}

In the following, the roaming limits $\tilde{Q}_{r, l}$ resulting from the polynomials $Q_{5}^{1 / 2}$ are presented. Normalising them with $F_{1}=\frac{1 \pm i}{\sqrt{2}} e^{\theta_{0} / 4}$ and performing the CPT symmetrisation that eliminates the imaginary terms, they are found to be identical with (2.41):

$\tilde{Q}_{0,5}=\left(y_{1} y_{2} y_{3} y_{4} y_{5}\right)^{2}$

$\tilde{Q}_{1,4}=y_{1} y_{2} y_{3} y_{4}\left(\frac{1}{x_{1}^{2}} \pm \frac{i\left(y_{1}+y_{2}+y_{3}+y_{4}\right)}{x_{1}}\right)$

$\tilde{Q}_{2,3}=\frac{1+x_{1} x_{2}\left(y_{2} y_{3}+y_{1} y_{3}+y_{1} y_{2}\right) \pm i\left(x_{2} x_{1}^{2} y_{1} y_{2} y_{3}+x_{1}\left(y_{1}\left(x_{2}^{2} y_{2} y_{3}+1\right)+y_{2}+y_{3}\right)+x_{2}\left(y_{1}+y_{2}+y_{3}\right)\right)}{x_{1} x_{2}}$

$\tilde{Q}_{3,2}=\frac{1+x_{1} x_{2} y_{1} y_{2}+x_{1} x_{3} y_{1} y_{2}+x_{2} x_{3} y_{1} y_{2} \pm i\left(y_{1}+y_{2}\right)\left(x_{1}\left(x_{2} x_{3} y_{1} y_{2}+1\right)+x_{2}+x_{3}\right)}{y_{1} y_{2}}$

$\tilde{Q}_{4,1}=\frac{x_{1} x_{2} x_{3} x_{4}\left(1 \pm i\left(x_{1} y_{1}+x_{2} y_{1}+x_{3} y_{1}+x_{4} y_{1}\right)\right)}{y_{1}^{2}}$

$\tilde{Q}_{5,0}=x_{1}^{2} x_{2}^{2} x_{3}^{2} x_{4}^{2} x_{5}^{2}$.

From the following table, the asymptotic divergence of various parts of the sinh-Gordon form factors can be studied. The overall scaling is $e^{\theta_{0} / 4}$ again as predicted by (2.35).

\begin{tabular}{|l|c|c|c|c|}
\hline$n=5$ & Normalisation & $Q_{5}^{1 / 2}$ & Denominator & Overall scaling \\
\hline$r=0$ & $e^{-5 \theta_{0} / 4}$ & $e^{2 \theta_{0}}$ & 1 & $e^{3 \theta_{0} / 4}$ \\
\hline$r=1$ & $e^{3 \theta_{0} / 4}$ & $e^{4 \theta_{0}}$ & $e^{-4 \theta_{0}}$ & $e^{3 \theta_{0} / 4}$ \\
\hline$r=2$ & $e^{7 \theta_{0} / 4}$ & $e^{6 \theta_{0}}$ & $e^{-7 \theta_{0}}$ & $e^{3 \theta_{0} / 4}$ \\
\hline$r=3$ & $e^{7 \theta_{0} / 4}$ & $e^{8 \theta_{0}}$ & $e^{-9 \theta_{0}}$ & $e^{3 \theta_{0} / 4}$ \\
\hline$r=4$ & $e^{3 \theta_{0} / 4}$ & $e^{10 \theta_{0}}$ & $e^{-10 \theta_{0}}$ & $e^{3 \theta_{0} / 4}$ \\
\hline$r=5$ & $e^{-5 \theta_{0} / 4}$ & $e^{12 \theta_{0}}$ & $e^{-10 \theta_{0}}$ & $e^{3 \theta_{0} / 4}$ \\
\hline
\end{tabular}


Considering the limit of the polynomial $Q_{6}^{\tau}$ and multiplying them with the square root type factors we obtain the functions (3.17) apart from a sign which is cancelled by another minus sign from the normalisation.

$$
\begin{aligned}
& \tilde{Q}_{0,6}=-y_{1}^{3} y_{2}^{3} y_{3}^{3} y_{4}^{3} y_{5}^{3} y_{6}^{3} \\
& \tilde{Q}_{1,5}=-\frac{y_{1}^{2} y_{2}^{2} y_{3}^{2} y_{4}^{2} y_{5}^{2}}{x_{1}^{3}} \\
& \tilde{Q}_{2,4}=-\frac{y_{1} y_{2} y_{3} y_{4}\left(x_{1}^{2} x_{2}^{2} y_{1} y_{2} y_{3} y_{4}+x_{1} x_{2}\left(y_{3} y_{4}+y_{2}\left(y_{3}+y_{4}\right)+y_{1}\left(y_{2}+y_{3}+y_{4}\right)\right)+1\right)}{x_{1}^{2} x_{2}^{2}} \\
& \tilde{Q}_{3,4}=-\frac{x_{2} x_{3}\left(y_{2} y_{3}+y_{1}\left(y_{2}+y_{3}\right)\right)+x_{1}\left(x_{2}+x_{3}\right)\left(y_{2} y_{3}+y_{1}\left(y_{2}+y_{3}\right)\right)+1}{x_{1} x_{2} x_{3}} \\
& \tilde{Q}_{4,2}=-\frac{x_{3} x_{4} y_{1} y_{2}+x_{2}\left(x_{3}+x_{4}\right) y_{1} y_{2}+x_{1} y_{1} y_{2}\left(x_{2}\left(x_{3} x_{4} y_{1} y_{2}+1\right)+x_{3}+x_{4}\right)+1}{y_{1} y_{2}} \\
& \tilde{Q}_{5,1}=-\frac{x_{1} x_{2} x_{3} x_{4} x_{5}}{y_{1}^{2}} \\
& \tilde{Q}_{6,0}=-x_{1}^{2} x_{2}^{2} x_{3}^{2} x_{4}^{2} x_{5}^{2} x_{6}^{2} .
\end{aligned}
$$

The overall scaling of the form factors is identical with scaling of $F_{4}^{\tau}$ as shown in the following table:

\begin{tabular}{|c|c|c|c|c|c|}
\hline$n=6$ & Normalisation & $Q_{n}$ & Denominator & Square root & Overall scaling \\
\hline$r=0$ & $e^{-3 \theta_{0}}$ & $e^{3 \theta_{0}}$ & 1 & 1 & 1 \\
\hline$r=1$ & $e^{-\theta_{0} / 2}$ & $e^{5 \theta}$ & $e^{-5 \theta_{0}}$ & $e^{\theta_{0} / 2}$ & 1 \\
\hline$r=2$ & $e^{\theta_{0}}$ & $e^{7 \theta_{0}}$ & $e^{-9 \theta_{0}}$ & $e^{\theta_{0}}$ & 1 \\
\hline$r=3$ & $e^{3 \theta_{0} / 2}$ & $e^{9 \theta_{0}}$ & $e^{-12 \theta_{0}}$ & $e^{3 \theta_{0} / 2}$ & 1 \\
\hline$r=4$ & $e^{\theta_{0}}$ & $e^{11 \theta_{0}}$ & $e^{-14 \theta_{0}}$ & $e^{2 \theta_{0}}$ & 1 \\
\hline$r=5$ & $e^{-\theta_{0} / 2}$ & $e^{13 \theta_{0}}$ & $e^{-15 \theta_{0}}$ & $e^{5 \theta_{0} / 2}$ & 1 \\
\hline$r=6$ & $e^{-3 \theta_{0}}$ & $e^{15 \theta_{0}}$ & $e^{-15 \theta_{0}}$ & $e^{3 \theta_{0}}$ & 1 \\
\hline
\end{tabular}

Open Access. This article is distributed under the terms of the Creative Commons Attribution License (CC-BY 4.0), which permits any use, distribution and reproduction in any medium, provided the original author(s) and source are credited.

\section{References}

[1] A.B. Zamolodchikov, Resonance factorized scattering and roaming trajectories, J. Phys. A 39 (2006) 12847 [inSPIRE].

[2] M. Lassig, Multiple crossover phenomena and scale hopping in two-dimensions, Nucl. Phys. B 380 (1992) 601 [hep-th/9112032] [INSPIRE].

[3] M.J. Martins, Renormalization group trajectories from resonance factorized $S$ matrices, Phys. Rev. Lett. 69 (1992) 2461 [hep-th/9205024] [INSPIRE].

[4] P.E. Dorey and F. Ravanini, Staircase models from affine Toda field theory, Int. J. Mod. Phys. A 8 (1993) 873 [hep-th/9206052] [INSPIRE]. 
[5] M.J. Martins, Analysis of asymptotic conditions in resonance functional hierarchies, Phys. Lett. B 304 (1993) 111 [INSPIRE].

[6] P. Dorey and F. Ravanini, Generalizing the staircase models, Nucl. Phys. B 406 (1993) 708 [hep-th/9211115] [INSPIRE].

[7] P. Dorey, G. Siviour and G. Takács, Form factor relocalisation and interpolating renormalisation group flows from the staircase model, JHEP 03 (2015) 054 [arXiv: 1412.8442] [INSPIRE].

[8] A.B. Zamolodchikov, Irreversibility of the flux of the renormalization group in a $2 D$ field theory, JETP Lett. 43 (1986) 730 [Pisma Zh. Eksp. Teor. Fiz. 43 (1986) 565] [inSPIRE].

[9] J.L. Cardy, The central charge and universal combinations of amplitudes in two-dimensional theories away from criticality, Phys. Rev. Lett. 60 (1988) 2709 [INSPIRE].

[10] A.B. Zamolodchikov, Two point correlation function in scaling Lee-Yang model, Nucl. Phys. B 348 (1991) 619 [INSPIRE].

[11] A. Fring, G. Mussardo and P. Simonetti, Form-factors for integrable Lagrangian field theories, the sinh-Gordon theory, Nucl. Phys. B 393 (1993) 413 [hep-th/9211053] [INSPIRE].

[12] C. Ahn, G. Delfino and G. Mussardo, Mapping between the sinh-Gordon and Ising models, Phys. Lett. B 317 (1993) 573 [hep-th/9306103] [INSPIRE].

[13] G. Delfino, G. Mussardo and P. Simonetti, Correlation functions along a massless flow, Phys. Rev. D 51 (1995) 6620 [hep-th/9410117] [InSPIRE].

[14] G. Delfino, P. Simonetti and J.L. Cardy, Asymptotic factorization of form-factors in two-dimensional quantum field theory, Phys. Lett. B 387 (1996) 327 [hep-th/9607046] [INSPIRE].

[15] P. Fendley, H. Saleur and A.B. Zamolodchikov, Massless flows. 1. The sine-Gordon and $O(n)$ models, Int. J. Mod. Phys. A 8 (1993) 5717 [hep-th/9304050] [InSPIRE].

[16] P. Fendley, H. Saleur and A.B. Zamolodchikov, Massless flows. 2. The exact $S$ matrix approach, Int. J. Mod. Phys. A 8 (1993) 5751 [hep-th/9304051] [INSPIRE].

[17] B. Berg, M. Karowski and P. Weisz, Construction of Green functions from an exact $S$ matrix, Phys. Rev. D 19 (1979) 2477 [INSPIRE].

[18] A.N. Kirillov and F.A. Smirnov, A representation of the current algebra connected with the SU(2) invariant Thirring model, Phys. Lett. B 198 (1987) 506 [INSPIRE].

[19] F.A. Smirnov, Form factors in completely integrable models of quantum field theory, Adv. Ser. Math. Phys. 14 (1992) 1 [INSPIRE].

[20] A.E. Arinshtein, V.A. Fateev and A.B. Zamolodchikov, Quantum $S$ matrix of the (1+1)-dimensional Todd chain, Phys. Lett. B 87 (1979) 389 [INSPIRE].

[21] A. Koubek and G. Mussardo, On the operator content of the sinh-Gordon model, Phys. Lett. B 311 (1993) 193 [hep-th/9306044] [INSPIRE].

[22] S.L. Lukyanov and A.B. Zamolodchikov, Exact expectation values of local fields in quantum sine-Gordon model, Nucl. Phys. B 493 (1997) 571 [hep-th/9611238] [INSPIRE].

[23] G. Delfino and G. Mussardo, The spin spin correlation function in the two-dimensional Ising model in a magnetic field at $T=T_{c}$, Nucl. Phys. B 455 (1995) 724 [hep-th/9507010] [INSPIRE]. 
[24] P.H. Ginsparg, Applied conformal field theory, in Fields, strings and critical phenomena, Proceedings of the Les Houches Summer School, E. Brezin and J. Zinn-Justin eds., France June 28-August 51988 [hep-th/9108028] [INSPIRE].

[25] N. Reshetikhin and F. Smirnov, Hidden quantum group symmetry and integrable perturbations of conformal field theories, Commun. Math. Phys. 131 (1990) 157 [InSPIRE].

[26] V.P. Yurov and A.B. Zamolodchikov, Correlation functions of integrable 2D models of relativistic field theory: Ising model, Int. J. Mod. Phys. A 6 (1991) 3419 [InSPIRE].

[27] A.B. Zamolodchikov, Thermodynamic Bethe ansatz for RSOS scattering theories, Nucl. Phys. B 358 (1991) 497 [INSPIRE].

[28] F. Ravanini, R. Tateo and A. Valleriani, Dynkin TBAs, Int. J. Mod. Phys. A 8 (1993) 1707 [hep-th/9207040] [INSPIRE]. 